\title{
A review of pressure transient analysis in reservoirs with natural fractures, vugs and/or caves
}

\author{
Isah Mohammed ${ }^{1} \cdot$ Teslim O. Olayiwola $^{1} \cdot$ Murtadha Alkathim $^{1} \cdot$ Abeeb A. Awotunde $^{1} \cdot$ Saad F. Alafnan $^{1}$
}

Received: 24 September 2019 / Published online: 22 September 2020

(c) The Author(s) 2020

\begin{abstract}
A review of the pressure transient analysis of flow in reservoirs having natural fractures, vugs and/or caves is presented to provide an insight into how much knowledge has been acquired about this phenomenon and to highlight the gaps still open for further research. A comparison-based approach is adopted which involved the review of works by several authors and identifying the limiting assumptions, model restrictions and applicability. Pressure transient analysis provides information to aid the identification of important features of reservoirs. It also provides an explanation to complex reservoir pressuredependent variations which have led to improved understanding and optimization of the reservoir dynamics. Pressure transient analysis techniques, however, have limitations as not all its models find application in naturally fractured and vuggy reservoirs as the flow dynamics differ considerably. Pollard's model presented in 1953 provided the foundation for existing pressure transient analysis in these types of reservoirs, and since then, several authors have modified this basic model and come up with more accurate models to characterize the dynamic pressure behavior in reservoirs with natural fractures, vugs and/or caves, with most having inherent limitations. This paper summarizes what has been done, what knowledge is considered established and the gaps left to be researched on.
\end{abstract}

Keywords Natural fractures $\cdot$ Vugs $\cdot$ Caves $\cdot$ Pressure transient analysis

$\begin{array}{ll}\text { List of symbols } \\ B & \text { Fracture depth } \\ C & \text { Gas concentration }\left(\mathrm{m}^{3} / \mathrm{mol}\right) \\ c & \text { Compressibility } \\ c_{1} & \text { Liquid of compressibility } \\ C_{\mathrm{D}_{\mathrm{f}}} & \text { Dimensionless fracture conductivity } \\ c_{\mathrm{m}} & \text { Matrix compressibility coefficient } \\ c_{\mathrm{p}} & \text { Shale compressibility }\left(\mathrm{MPa}^{-1}\right)\end{array}$

Edited by Yan-Hua Sun

Abeeb A. Awotunde

awotunde@kfupm.edu.sa

Isah Mohammed

isah.mohammed@ymail.com

Teslim O. Olayiwola

olayiwolateslim9@gmail.com

Murtadha Alkathim

g201702990@kfupm.edu.sa

Saad F. Alafnan

safnan@kfupm.edu.sa

1 College of Petroleum Engineering and Geosciences, King

Fahd University of Petroleum and Minerals, Dhahran,

Kingdom of Saudi Arabia
$D_{\mathrm{f}} \quad$ Fractal wall roughness dimension (dimensionless)

$D_{\mathrm{K}} \quad$ Knudsen diffusion coefficient $\left(\mathrm{m}^{2} / \mathrm{s}\right)$

$g_{\mathrm{c}} \quad$ Conversion factor

$h \quad$ Reservoir thickness

$J_{\mathrm{K}} \quad$ Knudsen diffusion flux $\left[\mathrm{mol} /\left(\mathrm{m}^{2} \mathrm{~s}\right)\right]$

$J_{\mathrm{v}} \quad$ Viscous flow flux $\left[\mathrm{mol} /\left(\mathrm{m}^{2} \mathrm{~s}\right)\right]$

$K_{2} \quad$ Fracture permeability

$K_{\infty} \quad$ Viscous flow permeability $\left(\mathrm{m}^{2}\right)$

$K_{\text {app }} \quad$ Apparent permeability

$K_{\mathrm{K}} \quad$ Knudsen diffusion apparent permeability

$K_{\mathrm{v}} \quad$ Apparent viscous flow permeability

$L \quad$ Transport distance

$M \quad$ Gas molar mass $(\mathrm{kg} / \mathrm{mol})$

$P \quad$ Pressure $(\mathrm{Pa})$

$P_{0} \quad$ Initial pressure

$P_{\mathrm{D}} \quad$ Dimensionless pressure

$P_{\mathrm{f}} \quad$ Fracture pressure

$P_{\mathrm{L}} \quad$ Langmuir pressure (MPa)

$P_{\mathrm{m}} \quad$ Matrix pressure

$q \quad$ Flow rate per unit length

$q^{\text {well }}$ Well flow rate

$R \quad$ Universal gas constant $[\mathrm{J} /(\mathrm{mol} \mathrm{K})]$

$R \quad$ Pore radius (m)

$r \quad$ Reservoir radius 
$r_{\mathrm{w}} \quad$ Wellbore radius

$S_{\mathrm{D}_{\mathrm{f}}} \quad$ Dimensionless fracture storage

$S_{\mathrm{m}} \quad$ Mechanical skin

$S_{\mathrm{S}_{\mathrm{f}}} \quad$ Dimensionless fracture skin

$T \quad$ Temperature (K)

$t \quad$ Time

$t_{\mathrm{D}} \quad$ Dimensionless time

$V_{\mathrm{L}} \quad$ Langmuir volume $\left(\mathrm{m}^{3} / \mathrm{t}\right)$

$V_{\text {std }} \quad$ Mole volume of gas at standard constant $\left(\mathrm{m}^{3} / \mathrm{mol}\right)$

$w_{\mathrm{f}} \quad$ Fracture width

$X_{\mathrm{D}} \quad$ Dimensionless fracture length

$X_{\mathrm{f}} \quad$ Fracture half-length

$\frac{\mathrm{d} p}{\mathrm{~d} L} \quad$ Pressure gradient

\section{Greek}

$\alpha \quad$ Rarefaction coefficient

$\alpha \quad$ Fissured medium characteristics

$\beta \quad$ Fracture volume

$\delta \quad$ Fracture thickness

$\lambda \quad$ Gas molecule mean free path (m)

$\lambda \quad$ Warren and Root reservoir heterogeneity level

$\mu \quad$ Absolute viscosity

$\mu_{\text {eff }} \quad$ Viscosity with rarefaction effect

$\tau \quad$ Tortuosity, dimensionless

$\rho_{\text {s }} \quad$ Shale density $\left(\mathrm{t} / \mathrm{m}^{3}\right)$

$\phi \quad$ Porosity (dimensionless)

$\Phi \quad$ Flow potential

$\omega \quad$ Warren and Root fluid flow capacity

$\omega \quad$ Storativity ratio

\section{Introduction}

To successfully carry out flooding or enhanced oil recovery process, a good understanding of the reservoir fluid system and petrophysical characteristics of the rocks is desired. Most reservoir formations have a high degree of heterogeneity, and this usually results in reduced reliability of the reservoir information obtained from pressure transient analysis. Aside from the issues arising from the variation in petrophysical properties, for a long time, scientists have attempted to characterize these properties using the variation of pressure with time. This is based on the general belief that these properties are a function of time-dependent variables such as pressure. In highly heterogeneous formations such as naturally fractured and karst reservoirs, earlier methods of pressure transient analysis are expected to give unsatisfactory results because of the assumption of uniformity and homogeneity of reservoir pore structure.

Fractured reservoirs are known to be heterogeneous, with openings (fractures and fissures) of varying sizes which leads to the creation of vugs and interconnected channels within a reservoir. Fluid storage is mostly in the porous block of the reservoir which is characterized by low permeability, whereas the fracture has high permeability and low storage capacity. These systems have undergone extensive studies, and this work presents a critical review of such works to provide insights into what knowledge has been gained thus far and what limitations are inherent in the existing models and how best to improve them. Pollard was one of the first persons to publish a study in 1953 on pressure transient analysis, and in 1959, the first pressure transient analysis (PTA) models were made available for well test interpretation from a two-porosity system (Pollard 1959). Since then, the behavior of fractured reservoirs along with pressure transient analysis in such mediums has become a highly researched area in the field of reservoir engineering. Thus, this work will state all the models presented thus far and analyze their scope of coverage in predicting pressure changes in fractured mediums. The work is divided into several subheadings to enable clear comparison and analysis of the various sections considered.

\section{Naturally fractured reservoirs}

Fractures have different definitions depending on the point of view. However, from a geomechanics point of view, a fracture is said to be a surface phenomenon that could be vertical or horizontal and is caused by the loss of cohesion in the texture of a rock. Fracturing involves the formation of joints and fissures when rocks break up. Fractures are caused by stresses and are termed natural or induced depending on whether it was created purposely. Naturally fractured formations are those characterized by secondary porosities in addition to primary porosity (Gong and Rossen 2017; Odeh 1965, 1959). The induced porosity (secondary porosity) results from stresses or tensional forces that cause brittle formations to crack. Figure 1 shows a three-layered reservoir with layers labeled $a, b$ and $c$. The layer with the weakest bonds (cohesion) would be the first to be fractured. Thus, in Fig. 1, layer $b$ has weak cohesion and it is thus fractured while layers $a$ and $c$ can withstand further stress.

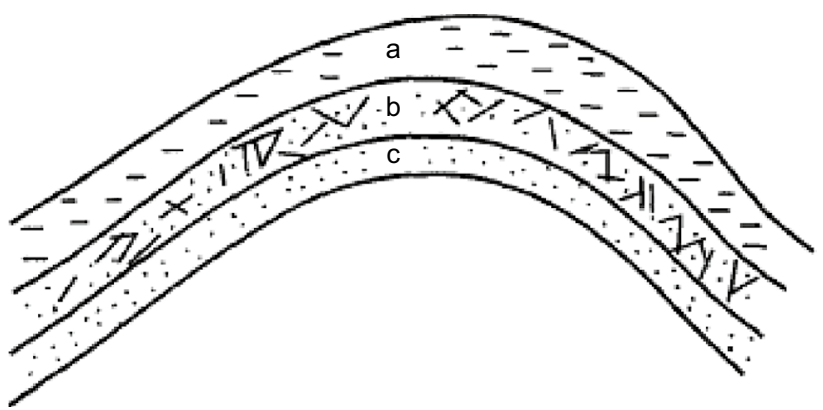

Fig. 1 Three-layered reservoir (van Golf-Racht 1982) 
Naturally fractured reservoirs have been identified as some of the highly productive oil and gas reservoirs. Many fields containing naturally fractured reservoirs saw significant hydrocarbon production mainly during their primary recovery phases. Examples of such fields include the Asmari Formation in Iran (Alsharhan and Nairn 1997; Levorsen and Berry 1967), Ekofisk Maastrichtian chalk in the North Sea (Fritsen and Corrigan 1990; Owen and Thomas 2002), Gianitic Basement Formation (Areshev et al. 1992; Tandom et al. 1999), Permian Formation (Wilkinson and Hammond 1990) and Monterey Formation (Luthi 2001). In the majority of the studies involving these fields, the fracture networks were observed to have formed as a result of shearing and tensile forces acting on the rocks due to tectonic actions (Luthi 2001).

The origin of fractures has long been a topic of debate among researchers and professionals especially concerning its intensity and reservoir trapping significance (van GolfRacht 1982). Friedman et al. (Friedman et al. 1976) classified fractures into two classes: those related to regional fractures and those related to folding. However, Hodgson (1961) had earlier postulated that there exists no genetic relationship between fold and fractures and asserted that joints are formed as a result of fatigue in the early diagenesis stage. Price (1966), on the other hand, asserted that even though joint could have been formed in the early stage of the diagenesis process as claimed by Hodgson (1961), it is impossible for it to have survived post-depositional compaction. Cook and Johnson (1970) concluded that joints could survive late diagenetic activities such as compaction, consolidation and burial. However, based on the analysis of fracture density and layer thickness by Harris et al. (1960), the authors affirmed the relationship between the two and it is safe to say, the origins of fractures could be structural and nonstructural related.

Due to the varying geological and structural configuration of various naturally fractured reservoirs, researchers have made several attempts at classifying them into different types with similar characteristics. Notably, van GolfRacht (1982), Allan and Sun (2003), Bourbiaux (2010), Nelson (2001), Ng and Aguilera (1991) and others have attempted to use wide-ranging definitions with some similarities. The most acceptable definition is based on the relationship between fluid storage and flow capacity. The type of naturally fractured reservoirs (Fig. 2) identified under this category includes

(A) Type 1: This type of formation has little or no matrix porosity and permeability. Here, the contribution of the matrix to flow could be negligibly small compared to that of fractures which serve as the primary pathway and storage for the reservoir fluid. An example of such

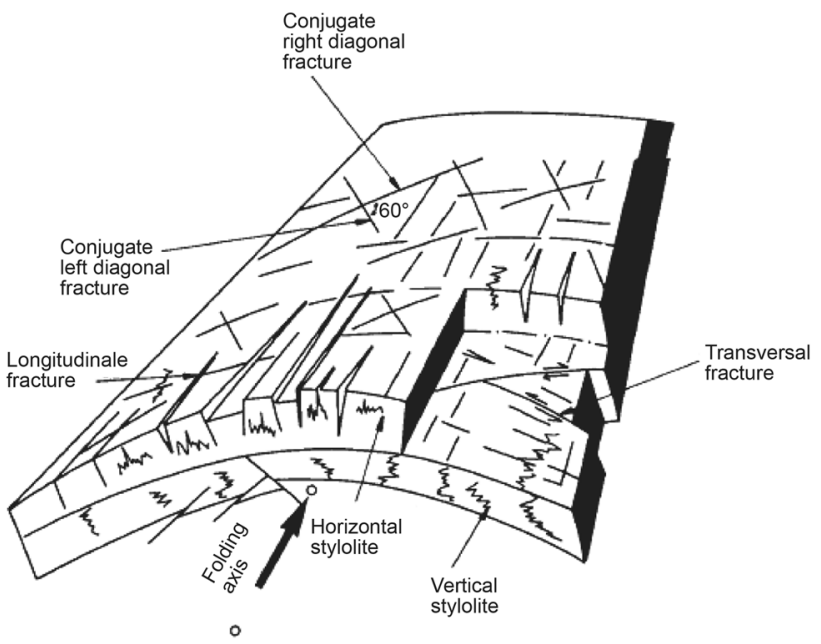

Fig. 2 Types of fractures as a result of folding (van Golf-Racht 1982)

formation can be found in the Keystone field in Texas with a characteristic average porosity of about $20 \%$ (Firoozabadi 2000).

(B) Type 2: This type of reservoir has low matrix porosity and permeability with formation fluid flow due to the intensity of the fractures and its distribution dictating the production. An example of this type of formation can be found in Asmari limestone reservoirs with about 10\%-20\% matrix porosity (Saidi 1987).

(C) Type 3: This type of reservoir has high matrix porosity and low permeability. Here, the bulk of the fluids are stored in the matrix. The pore volume of fractures is small compared to that of the matrix. Also, the flow capacity of the reservoir is fracture-dominated and this type of reservoir is commonly found in the North sea Ekofisk chalk formation with an average matrix porosity of about 35\% (Hermansen et al. 1997).

(D) Type 4: This type of formation is characterized by high matrix porosity and permeability with no additional porosity contribution from the fractured medium. In this type of reservoir, the fracture contributes less to the total available fluid flow and this typically enhanced permeability leads to increased anisotropy rather than improved fluid flow.

\section{Model development}

The first fractured reservoir was discovered in 1880 (Willis and Hubbert 1955). However, the well-known well test analysis methods and models were not available until the 1950s and even at that, models were only available for homogeneous formations. One of the most practical 
models recognized in the literature was presented by Horner (1951), which produced graphs of wellbore flowing pressures and logarithmic time called Horner time $\left(t_{\mathrm{p}}+\mathrm{d} t\right) / \mathrm{d} t$. However, this plot does not apply to formations with fractures. Based on the type of fissures found in the Middle East limestone reservoirs, Baker (1955) proposed a method of determining the sizes of the fissures and volume of fluid in a reservoir. The concept of fluid flow between two parallel plates was utilized as given by Lamb (1945) and Huitt (1956). The flow through such setup is given as

$q=-\frac{b \omega_{\mathrm{f}}^{2}}{12 \mu} \frac{\mathrm{d} p}{\mathrm{~d} L}$

where $q$ is the flow rate per unit length, $\omega_{\mathrm{f}}$ is the fracture width, $b$ is the fracture depth, $\mu$ is the fluid viscosity and $\mathrm{d} p / \mathrm{d} L$ is the pressure gradient across the flow interval of length $L$.

\subsection{Pressure transient analysis for fractured mediums}

In describing the pressure transient analysis in a fractured medium, various modeling approaches have been applied. In the models, attempts have been made to account for the distinct porosity types within the formation by dividing the formation into two regions: that containing only formation matrix and the other containing only fractures. In this section, we present the various attempts at modeling the fluid flow through a naturally fractured reservoir.

Naturally fractured reservoirs have been characterized to exhibit two distinct forms of reservoir porosity types: matrix porosity and fracture porosity (Kazemi 1969; de Swaan 1976; Warren and Root 1963). The matrix region is a region having a high percentage of fine pores with characteristically high storage but low flow capability. The fracture region has a higher flow capacity but lower storage capacity as compared to the matrix region. Most studies aimed at characterizing flow in a naturally fractured reservoir typically consider the formation as having two distinct regions. One early work on flows in fractured formations was that carried out by Pollard (1959), in which the author evaluated the flow behavior of acid treatment operation using pressure transient analysis. Pollard proposed a method based on classifying naturally fractured reservoirs as being delineated into three regions, namely rock matrix, fracture and the damaged/improved region close to the wellbore. Further, Pollard posited that fluid flows typically from a high porosity region to the highly permeable fractures (Fig. 3), eventually reaching the wellbore through the damaged/improved region and that there exists no contact between the high porous region and the wellbore.

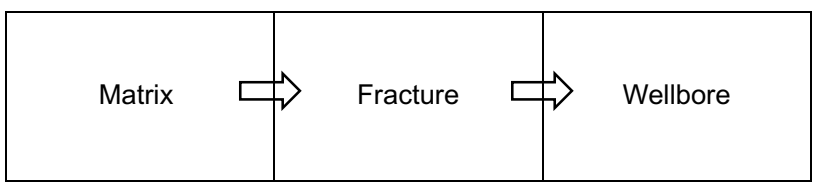

Fig. 3 Flow pattern in fractured reservoir

Also, the author observed that for all described regions, the average buildup pressure is approximately defined in the form of exponential time decay with different decay coefficients with each region occurring successively during the pressure buildup test. Pollard (1959) proposed an equation with three distinct terms each representing the pressure transient due to flow as shown in their earlier observed three distinct regions:

$$
\begin{aligned}
\left(\bar{p}-p_{\mathrm{w}}\left(\Delta t_{\mathrm{AD}}\right)\right)= & C_{\mathrm{D}} \exp \left[-\alpha_{1 \mathrm{D}} \pi r_{\mathrm{eD}}^{2} \Delta t_{\mathrm{AD}}\right]+D_{\mathrm{D}} \exp \left[-\alpha_{2 \mathrm{D}} \pi r_{\mathrm{eD}}^{2} \Delta t_{\mathrm{AD}}\right] \\
& +\left(p-p_{\mathrm{w}}-C-D\right)_{\mathrm{D}} \exp \left[-\alpha_{3 \mathrm{D}} \pi r_{\mathrm{eD}}^{2} \Delta t_{\mathrm{AD}}\right]
\end{aligned}
$$

where $C_{\mathrm{D}}$ and $D_{\mathrm{D}}$ are the decay coefficients. This equation is applicable to estimate properties such as wellbore damage and fracture volume. A pseudo-steady-state assumption was made as well as two types of the void (fine voids and coarse void) during the mathematical development, which according to Dikkers (1964) found application in the La Paz field at that time. Barenblatt et al. (1960) laid the foundation of the physical principles of the mathematical modeling of fractured mediums based on the interaction between the matrix region and the fracture region. This interaction is expressed as Barenblatt et al. (1960)

$q_{\mathrm{mf}}=\rho \alpha\left(p_{\mathrm{m}}-p_{\mathrm{f}}\right)$

where $q_{\mathrm{mf}}$ is the flow rate from the matrix to fracture, $\alpha$ is the fissured medium characteristic parameter, $p_{\mathrm{m}}$ is the pressure in the matrix and $p_{\mathrm{f}}$ is the pressure in the fracture.

Applying the continuity equation and Darcy's law and assuming a slightly compressible liquid of compressibility $c_{1}$ and matrix compressibility coefficient $c_{\mathrm{m}}$, Barenblatt et al. (1960) came up with a pressure diffusivity equation relating the matrix and fracture as

$\frac{\partial p_{\mathrm{f}}}{\partial t}-\frac{k_{\mathrm{f}}}{\alpha} \frac{\partial \Delta p_{\mathrm{f}}}{\partial t}=\frac{k_{\mathrm{f}}}{\mu\left(c_{\mathrm{m}}+\phi_{\mathrm{m}} c_{1}\right)} \Delta p_{\mathrm{f}}$

Pirson and Pirson (1961) extended Pollard's approach to estimating the volume of the matrix porosity and radius of well influence. In a further study developed for an infinite reservoir system, Warren and Root (1963) applied an approach different from earlier works by Pollard (1959) and Pirson and Pirson (1961). Here, the authors assumed that the reservoir pore system is having primary and secondary porosities. The primary porosity is composed of a set of rectangular building blocks called parallelepipeds which 
are homogeneous, isotropic and identical. The secondary porosity included in the coarse features with a uniform set of continuous fractures, each fraction having a property parallel to a principal axis of permeability. Warren and Root (1963)'s model depicts an overlapping arrangement of matrix and fractures (Fig. 4) with a quasi-steady-state flow assumed within the matrix at small times and unsteady-state flow within the fractures. The authors assumed a quasi-steadystate flow within the matrix at small times and unsteadystate flow within the fractures. The authors concluded that the behavior of this reservoir type will produce two parallel straight lines (Fig. 5) representing early and late times connected by a transition curve. These pressure signatures are controlled by two properties, $\omega$, a measure of fluid flow capacity of the fracture porosity and $\lambda$, a measure of the level of reservoir heterogeneity.

Furthermore, Warren and Root (1963) presented a model utilizing the concept of mathematical superposition of two mediums as introduced by Barenblatt et al. (1960). The authors discussed the fluid flow equation by Barenblatt et al. (1960) that represents the flow in the matrix and fractures as represented in Eqs. (5)-(7).

$-\nabla \cdot\left(\rho \vec{u}_{\mathrm{f}}\right)+q^{\mathrm{mf}}=\frac{\partial\left(\phi_{\mathrm{f}} \rho\right)}{\partial t}$,

and

$-\nabla \cdot\left(\rho \vec{u}_{\mathrm{m}}\right)-q^{\mathrm{mf}}=\frac{\partial\left(\phi_{\mathrm{m}} \rho\right)}{\partial t}$

where $q^{\mathrm{mf}}$ is the pseudo-steady-state flow rate between the matrix and the fracture. $q^{\mathrm{mf}}$ is given by

$q^{\mathrm{mf}}=\rho \frac{\sigma k_{\mathrm{m}}}{\mu}\left(p_{\mathrm{f}}-p_{\mathrm{m}}\right)$

The authors obtained the diffusivity equations in one dimension in both dimensional form:
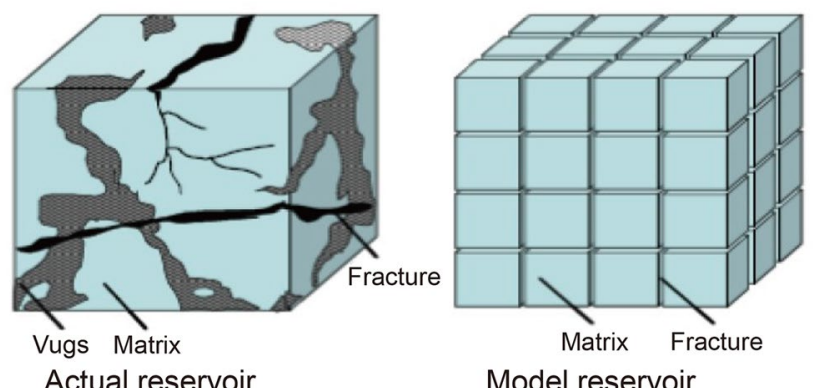

Fig. 4 Warren and Roots representation of fractured reservoirs (Warren and Root 1963)

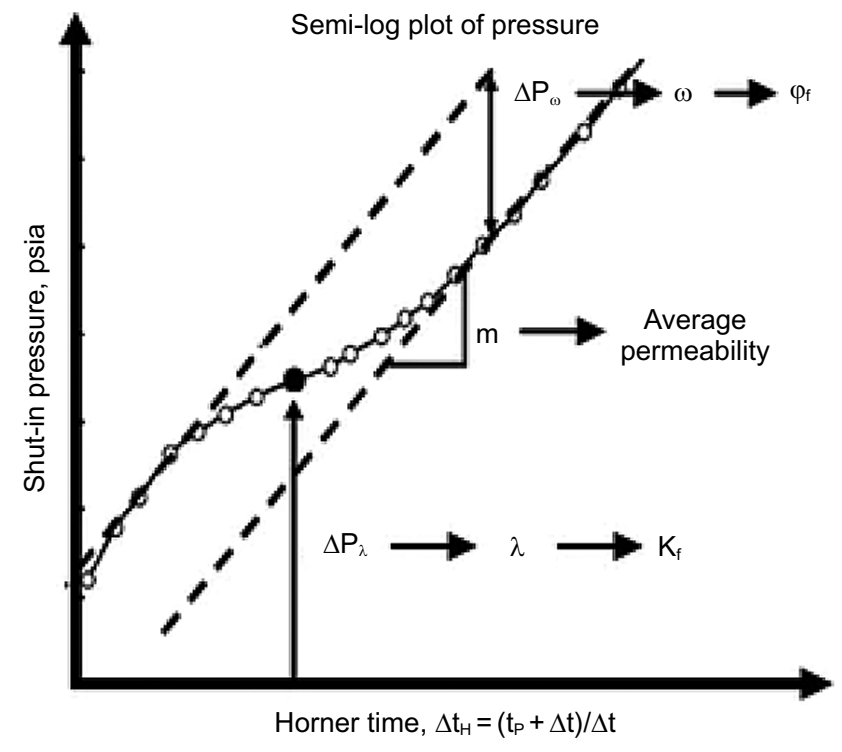

Fig. 5 Typical pressure buildup curve for Warren and Roots reservoir description (Bahrami et al. 2012)

$\frac{\sigma k_{\mathrm{m}}}{\mu}\left(p_{\mathrm{f}}-p_{\mathrm{m}}\right)+\phi_{\mathrm{m}} c_{\mathrm{m}} \frac{\partial p_{\mathrm{m}}}{\partial t}=0$

$\frac{k_{\mathrm{f}}}{\mu}\left[\frac{1}{r} \frac{\partial}{\partial r}\left(r \frac{\partial p_{\mathrm{f}}}{\partial r}\right)\right]+\phi_{\mathrm{f}} c_{\mathrm{f}} \frac{\partial p_{\mathrm{f}}}{\partial t}-\phi_{\mathrm{m}} c_{\mathrm{m}} \frac{\partial p_{\mathrm{m}}}{\partial t}=0$

and dimensionless form:

$(1-\omega) \frac{\partial p_{\mathrm{D}_{\mathrm{m}}}}{\partial t_{\mathrm{D}}}-\lambda\left(p_{\mathrm{D}_{\mathrm{f}}}-p_{\mathrm{D}_{\mathrm{m}}}\right)$,

$\frac{1}{r_{\mathrm{D}}} \frac{\partial}{\partial r_{\mathrm{D}}}\left(r_{\mathrm{D}} \frac{\partial p_{\mathrm{D}_{\mathrm{f}}}}{\partial r_{\mathrm{D}}}\right)-\omega \frac{\partial p_{\mathrm{D}_{\mathrm{f}}}}{\partial t_{D}}+(1-\omega) \frac{\partial p_{\mathrm{D}_{\mathrm{m}}}}{\partial t_{\mathrm{D}}}=0$.

The dimensionless variables in Eqs. (10) and (11) are given by

$r_{\mathrm{D}}=r / r_{\mathrm{w}}$,

$t_{\mathrm{D}}=\frac{k_{\mathrm{f}} t}{\left(\phi_{\mathrm{m}} c_{\mathrm{m}}+\phi_{\mathrm{f}} c_{\mathrm{f}}\right) \mu r_{\mathrm{w}}^{2}}$,

$p_{\mathrm{D}}=\frac{2 \pi r h}{\mu q}\left(p-p_{\mathrm{i}}\right)$,

$\lambda=\frac{\sigma k_{\mathrm{m}} r_{\mathrm{w}}^{2}}{k_{\mathrm{f}}}$, 
$\omega=\frac{\phi_{\mathrm{f}} c_{\mathrm{f}}}{\phi_{\mathrm{f}} c_{\mathrm{f}}+\phi_{\mathrm{m}} c_{\mathrm{m}}}$

The authors provided solutions to both finite-acting and infinite-acting reservoir systems. The dimensionless wellbore flow pressure for the infinite-acting reservoir was given as

$p_{\mathrm{D}_{\mathrm{f}_{\mathrm{w}}}}=\frac{1}{2}\left[0.80907+\ln t_{\mathrm{D}}+E i\left(-\frac{\lambda t_{\mathrm{D}}}{\omega(1-\omega)}\right)-E i\left(-\frac{\lambda t_{\mathrm{D}}}{1-\omega}\right)\right]$

This solution looks like the conventional solution to the single-porosity reservoir system with only a difference of $\Delta$ given by

$\Delta=\frac{1}{2}\left[E i\left(-\frac{\lambda t_{\mathrm{D}}}{\omega(1-\omega)}\right)-E i\left(-\frac{\lambda t_{\mathrm{D}}}{1-\omega}\right)\right]$

The solution to the finite-acting naturally fractured reservoir system is given by

$p_{\mathrm{D}_{f_{\mathrm{w}}}}=\left(\frac{2}{r_{\mathrm{D}_{\mathrm{e}}}^{2}}\right)\left[t_{\mathrm{D}}+\frac{(1-w)^{2}}{\lambda} \exp \left(-\frac{\lambda t_{\mathrm{D}}}{w(1-w)}\right)+\ln r_{\mathrm{D}_{\mathrm{e}}}-\frac{3}{4}+s\right]$

The difference between Eq. (14) and the solution to the single-porosity medium is given by

$\Delta=\frac{(1-w)^{2}}{\lambda} \exp \left(-\frac{\lambda t_{\mathrm{D}}}{w(1-w)}\right)$

Based on these solutions, Warren and Root (1963) obtained a plot with a characteristic of three different regions. In the plot, the early time is represented by a straight line defining that stage where fracture depletion dominates. A transition zone describes the effect of the matrix-dominated flow period while the late time depicts the period when both porosity types influence production. The significance of Warren and Root's model laid the foundation for future work on pressure transient analyses in naturally fractured reservoirs.

In another study, Odeh (1965) attempted to account for the failure of the Warren and Roots model in some field applications. In his research, he applied available field pressure data to mathematically analyze the behavior of reservoirs like that of Warren and Roots model but with an additional assumption of uniform flow capacity and degree of fracturing within the fractures typical of a reservoir with small grid block compared to the reservoir dimension. Odeh (1965)'s approach gave a general model expressed as

$$
\begin{aligned}
\Delta p= & \frac{-q \mu}{4 \pi k_{\mathrm{f}} h \beta}\left[\ln \left[\frac{4 t k_{\mathrm{f}} V_{\mathrm{f}}}{r^{2} \phi_{\mathrm{f}}\left(c_{\mathrm{f}} V_{\mathrm{f}}+c_{\mathrm{m}} V_{\mathrm{m}}\right)}\right]-0.5772-E i\left(-C_{\mathrm{t}}\right)\right. \\
& \left.+E i\left(-C_{\mathrm{t}}\left(1+\frac{V_{\mathrm{m}} c_{\mathrm{m}}}{V_{\mathrm{f}} c_{\mathrm{f}}}\right)\right)\right]
\end{aligned}
$$

Equation (16) can be approximated as

$\Delta p=\frac{-q \mu}{4 \pi k_{\mathrm{f}} h \beta}\left[E i\left(\frac{-r^{2} \mu\left(c_{\mathrm{f}} \beta \phi_{\mathrm{f}}+c_{\mathrm{m}}(1-\beta) \phi_{\mathrm{m}}\right)}{4 k_{\mathrm{f}} \beta t}\right)\right]$

Equation (17) has a form similar to Horner's buildup equation (Horner 1951) for a homogenous reservoir. His model has a characteristic property $\beta$ that defines the entire fracture volume as a function of the studied core volume.

Odeh (1965) observed that both the pressure buildup ( $p$ vs. $\ln [(t+\Delta t) / \Delta t]$ and pressure drawdown plots $(p$ vs. $\ln t)$ for a fractured reservoir give a characteristic straight-line plot similar to that of a homogenous reservoir. Thus, the author concluded that no specific model could be truly representative of every type of fractured reservoirs. In rebuttal within the same publication, Warren and Root observed that Odeh's model was misleading and that the new parameters by Odeh can be adjusted to that resulting in the same model as Warren and Roots.

Kazemi (1969) observed some limitations in the widely used Warren and Roots model. In his study, Kazemi considered a radial flow in a well located at the center of a finite circular reservoir with horizontal fracture orientation as shown in Fig. 6. The author replaced Warren and Root's assumption of quasi-steady-state flow with the unsteady-state flow and observed that there exists a considerable time difference between the disappearance time of the early-time plot in the Warren and Root model and the start of the quasi-steady state. This difference may result in a poor estimation of critical properties such as relative storage capacity. The author then concluded that for larger times, naturally fractured reservoirs tend to behave like a homogenous reservoir and that, the Warren and Roots model gives a substantially reasonable estimate but has limitations in formations with small fracture-matrix flow capacity difference. The differential equations of a reservoir undergoing unsteady-state flow with flow potential $\Phi$, fracture thickness $\delta$, fracture block thickness $h$, and compressibility $c$ are

$\frac{1}{r} \frac{\partial}{\partial r}\left(r \frac{\partial \Phi}{\partial r}\right)+\frac{\partial^{2} \Phi}{\partial z^{2}}=\frac{\phi_{\mathrm{m}} \mu c_{\mathrm{m}}}{k_{\mathrm{m}}} \frac{\partial \Phi}{\partial t}$ for $\frac{\delta}{2}<z<\frac{h}{2}, \quad r_{\mathrm{w}}<r<r_{\mathrm{e}}$

for flow in the matrix, and

$\frac{1}{r} \frac{\partial}{\partial r}\left(k_{\mathrm{f}} r \frac{\partial \Phi}{\partial r}\right)+\frac{k_{\mathrm{m}}}{\delta / 2}\left(\frac{\partial \Phi}{\partial z}\right)_{z=\delta^{+} / 2}=\phi_{\mathrm{f}} \mu c_{\mathrm{f}} \frac{\partial \Phi}{\partial t}$ for $0<\mathrm{z}<\frac{\delta}{2}, r_{\mathrm{w}}<r<r_{\mathrm{e}}$ 

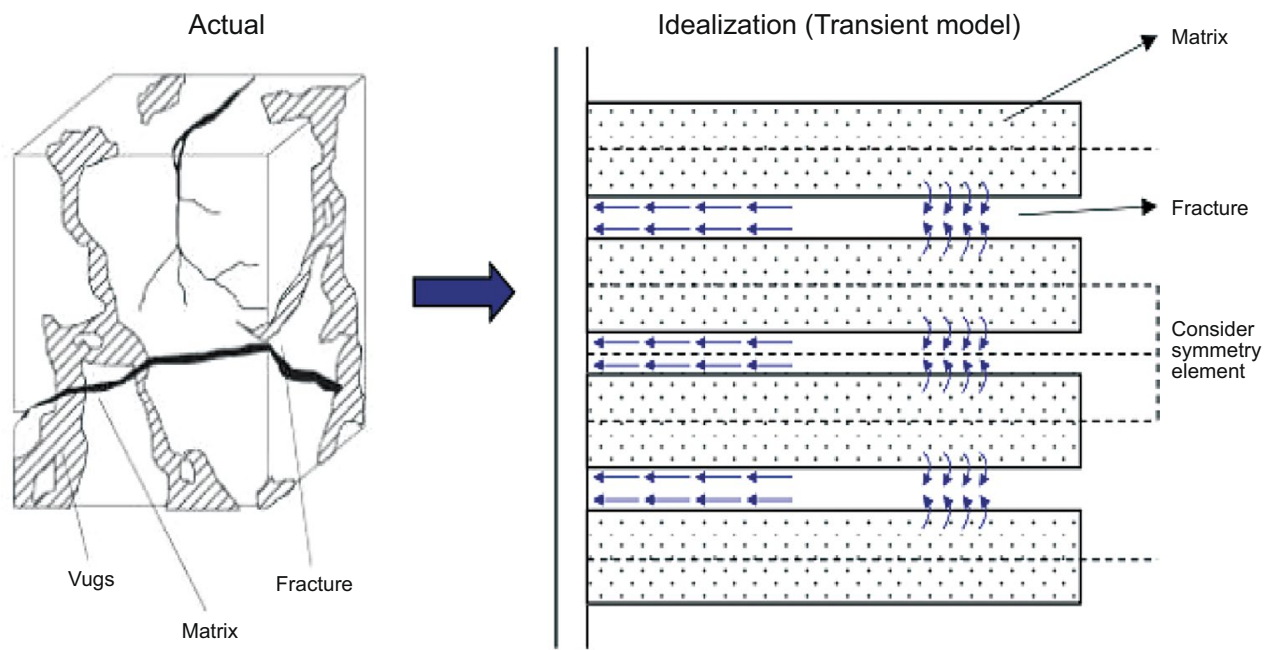

Fig. 6 Kazemi-fractured formation representation (Kazemi 1969)

for flow in the fracture. The flow potential is given by

$\boldsymbol{\Phi}=\rho(0)\left[\int_{0}^{p} \frac{\mathrm{d} p}{\rho(p)}+g z\right]$

de Swaan (1976) presented a transient state model for slightly compressible flow with a flow from the matrix into the fracture using two geometries. The author based his findings on the fact that matrix blocks can be modeled as rectangular solids whose transient pressure models are similar to those from heat flow theory. Using convolution theory, the flow between the matrix and fractured medium is given as

$q_{\mathrm{m}}=\frac{-2}{A_{\mathrm{m}} h_{\mathrm{f}}} \int_{0}^{t} \frac{\partial \Delta p_{\mathrm{f}}}{\partial r} q_{\mathrm{m}}(t-\tau) \partial \tau$

This term is substituted in the diffusivity equation and the pressure equations defining flow within the fractured medium was obtained. Here, for a fractured medium having a constant wellbore flowing rate and uniform pressure at the initial condition, the governing pressure equation is given as:

$\frac{k_{\mathrm{f}}}{\mu} \frac{\partial^{2} \Delta p_{\mathrm{f}}}{\partial r^{2}}=\phi_{\mathrm{f}} c \frac{\partial \Delta p_{\mathrm{f}}}{\partial r}-q_{\mathrm{m}}\left(\Delta p_{\mathrm{f}}, t\right)$

de Swaan (1976) provided a solution for early and long times without the transient period. Based on de Swans' theory, Najurieta (1980) presented all the solutions of the radial distances with time (Figs. 7, 8).

In a later attempt at getting a better insight into the flow behavior of a naturally fractured reservoir, other techniques such as pressure derivative solutions have been proposed (Bourdet et al. 1983; Escobar and Tiab 2002; Tiab 1994).

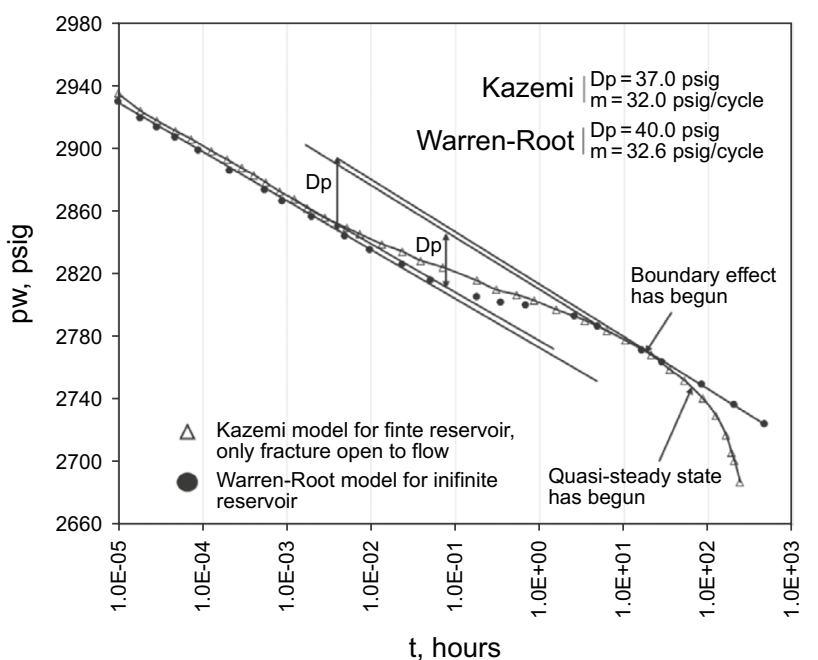

Fig. 7 Typical buildup curve for Kazemi model (Kazemi 1969)

This method gained prominence due to its ability to better capture the different flow regimes (Bourdet et al. 1983).

Engler and Tiab (1996) attempted to explore the benefits of applying derivative plots in fractured reservoirs. Here, the authors applied the Tiab direct synthesis (TDS) of a reservoir type with an idealized heterogeneous system of vugs, matrix and fractures. In modeling this, the authors assumed that the matrix element is separated by a uniformly continuous fractured region characterized by the pseudo-steady flow between it and the matrix as shown in Fig. 9. For a singlephase fluid flow, the pressure equation proposed by Warren and Root (1963) was employed. The authors obtained a pressure derivative equation for a formation with mechanical skin $\left(S_{\mathrm{m}}\right)$ as described by Eq. (23). 

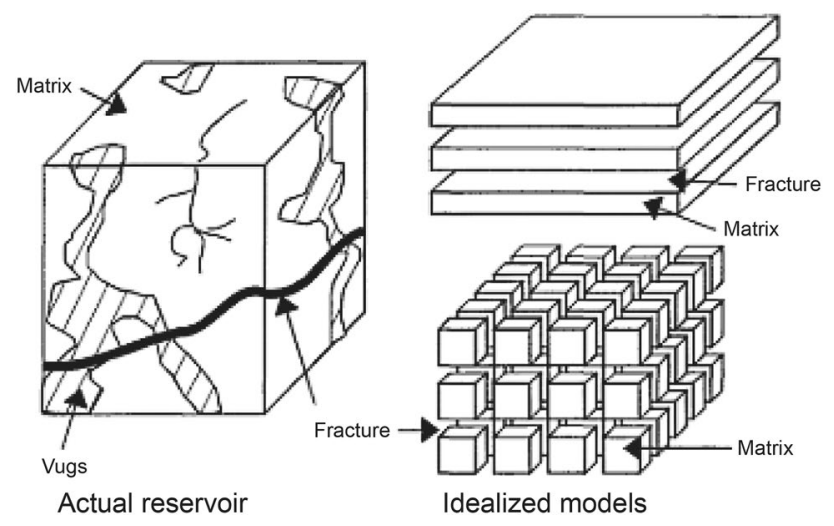

Fig. 8 The actual and idealized representation of the reservoir model by Engler and Tiab (1996)

$p_{\mathrm{D}_{\mathrm{w}}}^{\prime} t_{\mathrm{D}_{\mathrm{w}}}=\frac{1}{2}\left[1-\mathrm{e}^{-\frac{\lambda \mathrm{D}_{\mathrm{w}}}{1-w}}+\mathrm{e}^{-\frac{\lambda \mathrm{t}_{\mathrm{w}}}{w(1-w)}}\right]$

The terms in Eq. (23) have the following forms.

$p_{\mathrm{D}}=\frac{k_{\mathrm{f}} h \Delta p}{141.2 q \mu \beta_{\mathrm{o}}}$

$t_{\mathrm{D}_{\mathrm{w}}}=\frac{0.0002637 k_{\mathrm{f}} t}{\left(\phi_{\mathrm{m}} c_{\mathrm{m}}+\phi_{\mathrm{f}} c_{\mathrm{f}}\right) \mu r_{\mathrm{w}}^{2}}$

$\omega=\frac{\phi_{\mathrm{f}} c_{\mathrm{f}}}{\phi_{\mathrm{m}} c_{\mathrm{m}}+\phi_{\mathrm{f}} c_{\mathrm{f}}}$

$\lambda=\alpha r_{\mathrm{w}}^{2} \frac{k_{\mathrm{m}}}{k_{\mathrm{f}}}$

Equation (23) as presented by Engler and Tiab (1996) gives a characteristic curve for the undamaged and damaged

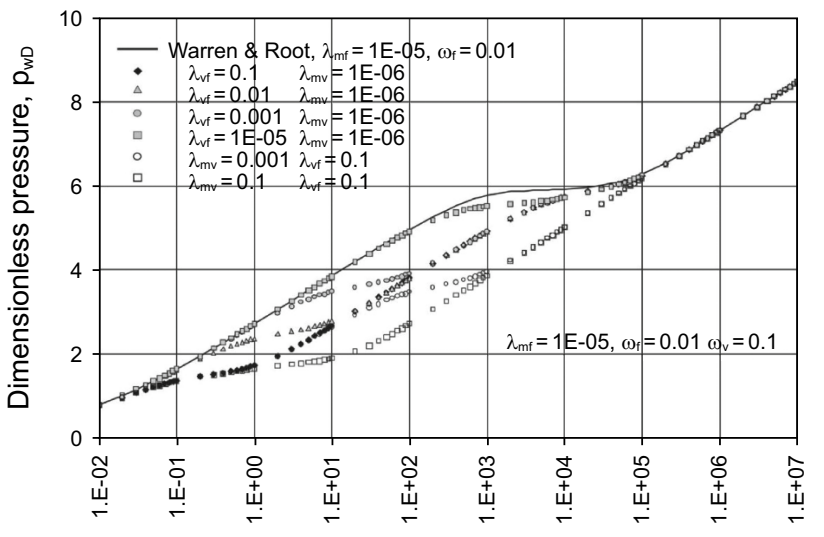

Dimensionless time, $t_{D}$

Fig. 10 Vuggy reservoir transient analysis (Velazquez et al. 2005)

reservoir as shown in Fig. 10. With this curve, various parameters representative of the reservoir formation can be estimated.

The infinite-acting flow period is represented by a horizontal straight line on the pressure derivative-type curve. This region defines the period where only the fracture contributes to production. The pressure derivative $\left(p_{\mathrm{Dw}}{ }^{\prime} \cdot t_{\mathrm{Dw}}\right)$ and fracture permeability $\left(k_{2}\right)$ during this period for a well experiencing no wellbore effect is given as

$p_{\mathrm{Dw}}^{\prime} t_{\mathrm{Dw}}=\frac{1}{2}$

$S_{\mathrm{m}}=\frac{1}{2}\left[\left(\frac{\Delta p}{\Delta p^{\prime} t}\right)_{r_{1}}-\ln \left(\frac{k_{\mathrm{f}} t_{r 1}}{s_{\mathrm{T}} \mu r_{\mathrm{w}}^{2}} \frac{1}{w}\right)+7.43\right]$

$k_{\mathrm{f}}=\frac{70.6 q \mu \beta_{\mathrm{o}}}{h\left(\Delta p^{\prime} t\right)}$

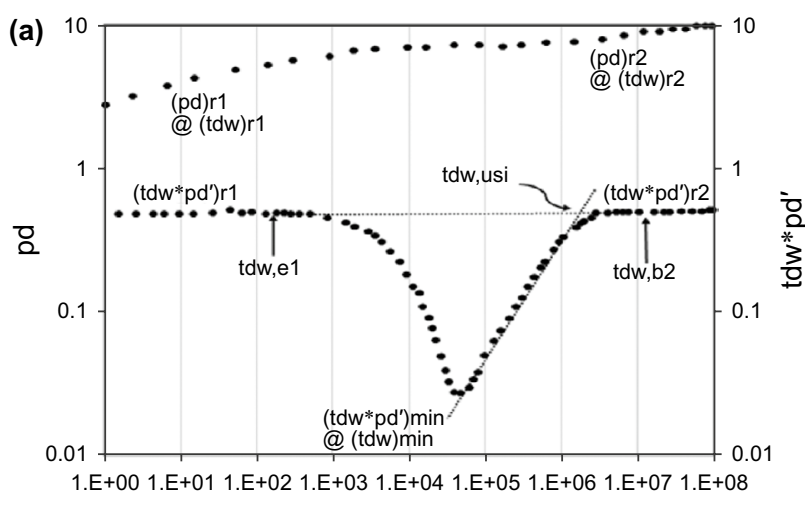

tdw

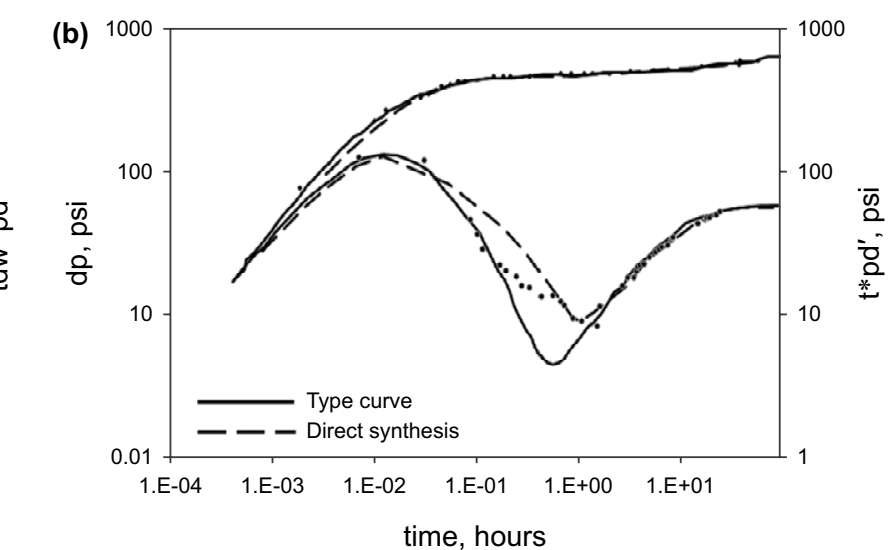

Fig. 9 Combined pressure and pressure derivative curve. a No skin or wellbore damage. b Skin damage (Engler and Tiab 1996) 
In a reservoir with a wellbore storage effect, the effect of early time represented by the straight line is nonexistent. After the early time, the transition period connects the early and late times. Here, the depth of the trough is dependent on the value of the Warren and Root storage coefficient $(w)$ but not on the interporosity parameter $(\lambda)$. The dimensionless terms depicting this timeline are defined as

$\omega=0.15866\left\{\frac{\left(\Delta p^{\prime} t\right)_{\min }}{\left(\Delta p^{\prime} t\right)_{\mathrm{r}}}\right\}+0.54653\left\{\frac{\left(\Delta p^{\prime} t\right)_{\min }}{\left(\Delta p^{\prime} t\right)_{\mathrm{r}}}\right\}^{2}$

$\lambda=\frac{42.5 h s_{\mathrm{T}} r_{\mathrm{w}}^{2}}{q \beta_{\mathrm{o}}}\left(\frac{\Delta p^{\prime} t}{t}\right)_{\text {min }}$

The late time of the Engler and Tiab (1996) pressure derivative plot exists in both the damaged and undamaged scenarios. Moreover, this period gives the idealistic representation of the mechanical skin $\left(S_{\mathrm{m}}\right)$ and the wellbore storage coefficient $(C)$.

$S_{\mathrm{m}}=\frac{1}{2}\left[\left(\frac{\Delta p}{\Delta p^{\prime} t}\right)_{r_{2}}-\ln \left(\frac{k_{\mathrm{f}} t_{r 2}}{s_{\mathrm{T}} \mu r_{\mathrm{w}}^{2}}\right)+7.43\right]$

$C=\left\{\begin{array}{l}\left(\frac{q \beta_{0}}{24}\right) \frac{t}{\Delta p} \\ \left(\frac{q \beta_{0}}{24}\right) \frac{t}{\Delta p^{\prime} * t}\end{array}\right.$

Rezk (2016) studied different pressure transient analysis techniques applicable in naturally fractured reservoirs. $\mathrm{He}$ considered PTA by Warren and Root (1963) model, Engler and Tiab (1996) and other types of type-curve matching. He concluded that the model proposed by Engler and Tiab offered various advantages compared to the conventional semi-log analysis by Warren and Root (1963). Most of the previous approaches at studying the behavior of fluid flow in naturally fractured reservoirs have strictly assumed no significant changes in the rock properties with pore pressure, effective stress and temperature (Berumen and Tiab 1997; Pedrosa 1986). As such these models are expected to fail when applied to a reservoir with stress-sensitive rock properties. Stress-dependent properties are typically associated with low-permeability formations, geopressured formations and fractured formations (Pedrosa 1986). Early works by various authors such as Cinco-Ley et al. (1985), Ostensen (1986), Pedrosa (1986), Raghavan et al. (1972), Samaniego et al. (1985) and Yilmaz et al. (1994) considered pressuredependent rock properties in pressure transient analysis for both conventional rocks and naturally fractured rocks. In most modeling approaches, these authors applied numerical techniques in obtaining solutions to both the linear and radial flow diffusivity equations under the assumption of either constant flowing wellbore pressure or constant well flow rate.

Pedrosa (1986) applied a form of the Cole-Hopf transformation to convert the nonlinear diffusivity equation into a linear diffusivity equation that can be solved using an analytical technique. The nonlinear diffusivity equation is expressed in a dimensional form in Eq. (35) and in a dimensionless form in (36), for a well producing at a constant well rate in an infinite-acting radial system. These equations are

$\frac{\partial^{2} p}{\partial r^{2}}+\frac{1}{r} \frac{\partial p}{\partial r}+\left(C_{\mathrm{L}}+\gamma\right)\left(\frac{\partial p}{\partial r}\right)^{2}=\frac{\phi \mu}{k}\left(C_{\mathrm{L}}+C_{\mathrm{m}}\right) \frac{\partial p}{\partial t}$

and

$\frac{\partial^{2} p_{\mathrm{D}}}{\partial r_{\mathrm{D}}^{2}}+\frac{1}{r_{\mathrm{D}}} \frac{\partial p_{\mathrm{D}}}{\partial r_{\mathrm{D}}}-\gamma_{\mathrm{D}}\left(\frac{\partial p_{\mathrm{D}}}{\partial r_{\mathrm{D}}}\right)^{2}=\mathrm{e}^{\gamma_{\mathrm{D}} p_{\mathrm{D}}} \frac{\partial p_{\mathrm{D}}}{\partial t_{\mathrm{D}}}$

In the final model, the author proposed a key parameter known as $\gamma_{D}$ to study the effect of pressure on the formation permeability. The reservoir model as described has an initial condition and inner and outer boundary conditions as expressed by Eqs. (37)-(39), respectively:

$p_{\mathrm{D}}\left(r_{\mathrm{D}}, t_{\mathrm{D}}=0\right)=0$

$\lim _{r_{\mathrm{D}} \rightarrow o}\left(r_{\mathrm{D}} \mathrm{e}^{-\gamma_{\mathrm{D}} p_{\mathrm{D}}} \frac{\partial p_{\mathrm{D}}}{\partial t_{\mathrm{D}}}\right)=-1$

$\lim _{r_{\mathrm{D}} \rightarrow \infty} p_{\mathrm{D}}\left(r_{\mathrm{D}}, t_{\mathrm{D}}\right)=0$

In Eqs. (37)-(39), the dimensionless terms are defined as

$$
\begin{aligned}
& r_{\mathrm{D}}=\frac{r}{r_{\mathrm{w}}}, \quad t_{\mathrm{D}}=\frac{k_{\mathrm{i}} t}{\phi_{\mathrm{i}} \mu\left(C_{\mathrm{L}}+C_{\mathrm{m}}\right) r_{\mathrm{w}}^{2}}, \\
& p_{\mathrm{D}}=\frac{2 \pi k_{\mathrm{i}} h}{q \mu}\left(p_{\mathrm{i}}-p\right), \quad \gamma_{\mathrm{D}}=\frac{q \mu}{2 \pi k_{\mathrm{i}} h} \gamma
\end{aligned}
$$

A limitation of the model as described by Pedrosa (1986) for flow in a stress-sensitive formation is the non-inclusion of properties representative of a naturally fractured formation. Berumen and Tiab (1997) studied the flow of a slightly compressible fluid of constant viscosity through a fracture located along the focal point/center of the reservoir. In the work, the authors assumed a high-velocity flow in both the fracture and formation. Such high-velocity flow could be represented by combining a Forchheimer transport equation

$\vec{u}=-\delta \frac{k(p)}{\mu} \nabla p$

with the continuity equation 
$-\nabla \cdot\left(\rho_{\mathrm{f}} \vec{u}\right)+\frac{q_{\mathrm{f}}(x, t)}{h}=\frac{\partial\left(\phi \rho_{\mathrm{f}}\right)}{\partial t}$

All the models presented so far have been for vertical welltypes. However, fields with naturally fractured reservoirs have since witnessed the use of horizontal wells. Horizontal wells have gained wide acceptance in the industry, especially in the development of unconventional reservoirs because it accelerates production, increases recovery and minimizes pressure drop along the wellbore. Chen et al. (2019) developed a semi-analytical model for the pressure transient analysis of fractured horizontal well with natural fracture networks. This work highlights the behavior of the pressure derivative in formations with both natural and hydraulic fractures and networks. The authors identified six flow regimes and provided a guide to analyzing fracture networks.

\subsection{Pressure transient analysis of vuggy reservoirs}

Vuggy reservoirs are composed of many reservoir bodies each of which may exhibit distinct fluid and pressure systems (Chen et al. 2017). These are features created as a result of dissolution processes that result in discontinuities within the reservoir (Barros-Galvis et al. 2015). Reservoir engineers have referred to these discontinuities as a type of pore system regardless of its origin. Vugs are a type of pore system and can be classified differently based on pore space genesis (Choquette and Pray 1970). Petrophysical properties as well as pore size distribution are also used to classify vugs within a rock. Vuggy pore space is classified into two: touching and separate vuggy pores. Touching vugs form an interconnected pore system while separate vugs have larger pore space than the particle size and are interconnected only through the interparticle pore network. The permeability of vuggy porous media depends on its interconnection of the pore space.

Literature has shown that the development of vuggy carbonate reservoirs has received attention for over 25 years, with most activities in China (Yao 2010). Shengeli and Tahe oil fields have been developed for over 15 years. There are significant fracture networks in these fields and water injections have been implemented in both fields to increase production. It is reported that the field recovery factor of these vuggy reservoirs is between $13 \%$ and $15 \%$ and has thus received less attention compared with the conventional oil reservoirs. Also, a rapid annual decline rate of $25 \%$ is experienced due to a lack of adequate understanding of the flow mechanics in such reservoirs. Xiong et al. (2016) provided an explanation of observations on the pressure derivative curves for the vuggy-fractured reservoirs via an experimental technique. This was to highlight the governing characteristics of fluid exchange in vuggy reservoirs and to highlight some theoretical claims in the literature. The model developed was tested and had a reasonable accuracy level. However, comparison with other existing techniques was not made. Moreover, the fluid-rock material exchange had been observed to affect flow characteristics. This phenomenon was however not captured by the authors.

Vuggy reservoirs from a geological viewpoint have experienced multiple processes including hydrocarbon accumulation, karst superposition and tectonic movements, etc. and are characterized by permeability anisotropy and heterogeneity. Additionally, this type of reservoir exhibits different porosity types and fluid flow mechanisms (cavity-free fluid flow and flow within the matrix), with a laminar flow regime in low-permeability matrices and turbulent flow in cavities (Li et al. 2019). The complicated fluid flow patterns within the matrix and cavity make the understanding of the flow mechanism difficult.

Vuggy reservoirs are identified to have the following characteristics.

1. Vuggy reservoirs exhibit multiple storage categories including fractures, vugs, cavities and matrix.

2. The scale of variation (anisotropy) is wide.

3. High heterogeneity as a result of post-diagenesis activities.

The above characteristics of the vuggy reservoir are responsible for the complexity of the flow mechanism resulting in difficulty in modeling such reservoirs. Due to multiple storage mediums, there exist dual and tripleporosity models for the vuggy reservoir system. Vuggy reservoirs are known to consist of both vugs and matrix systems. The physical properties of the formation matrix are considerably different from those of vugs. Also, the vugs are dispersed within the matrix system. Several models have been proposed for vuggy reservoirs considering vug geometries, pressure distributions and fluid flow mechanisms. One of the early works on naturally fractured reservoirs was presented by Warren and Root in 1963. However, an abnormal change in well pressure test slope over the transient period was observed in some reservoir transient pressure data. This slope change is due to the flow assumption of the homogenous matrix system. Because some naturally fractured reservoirs matrices can also be vuggy, a pseudo-steady-state triple-porosity model was proposed to describe the fluid flow in such reservoirs.

Traditional pressure transient analysis has been used as a method in the identification of different flow regimes and the determination of appropriate reservoir and fractures parameters. The presence of a complex geometric pore system poses challenges when applying conventional PTA in naturally fractured and vuggy reservoirs. In recent times, several authors have proposed modifications to existing PTA 
models to better characterize this complex pore system. Researchers such as Fuentes-Cruz et al. (2004) applied PTA to partially penetrating wells in a naturally fractured vuggy system. The authors observed that the presence of vugs and/ or caves may have a significant effect on the transient pres-
$-\lambda_{\mathrm{mv}}\left(p_{\mathrm{Dm}}-p_{\mathrm{Df}}\right)-\lambda_{\mathrm{mf}}\left(p_{\mathrm{Dm}}-p_{\mathrm{Df}}\right)=\left(1-\omega_{\mathrm{f}}-\omega_{\mathrm{v}}\right) \frac{\partial p_{\mathrm{Dm}}}{\partial t_{\mathrm{D}}}$

Furthermore, flow in the vugs is described by

$(1-\kappa) \frac{1}{r_{\mathrm{D}}} \frac{\partial}{\partial r_{\mathrm{D}}}\left(r_{\mathrm{D}} \frac{\partial p_{\mathrm{Dv}}}{\partial r_{\mathrm{D}}}\right)+\lambda_{\mathrm{mv}}\left(p_{\mathrm{Dm}}-p_{\mathrm{Dv}}\right)-\lambda_{\mathrm{vf}}\left(p_{\mathrm{Dv}}-p_{\mathrm{Df}}\right)=\omega_{\mathrm{v}} \frac{\partial p_{\mathrm{Dv}}}{\partial t_{\mathrm{D}}}$

sure and production curve behaviors. Fuentes-Cruz et al. (2004) studied the pressure behavior of naturally fractured vuggy reservoirs and the influence of secondary pore type on the shape of the production decline curve. The authors concluded that it is important to introduce the effect of the secondary porosity during type-curve matching. Guo et al. (2012) proposed a dual-permeability model to analyze the production behavior of a horizontal well in naturally fractured vuggy carbonate rocks. The authors assumed that the vugs are dispersed within the reservoir pore system implying the existence of only a two-pore system (matrix and fractures). They concluded that the type curve is strongly controlled by the interporosity flow characteristics, the existence of seven flow regimes for a constant-rate production and five flow regimes for a constant wellbore-pressure system. Nie et al. (2012) proposed a dual-porosity and dual-permeability approach to study flow behavior of a producing horizontal well in a naturally fractured reservoir formation. In contrast to the single-permeability modeling approach, the authors observed a significant difference in the type curve due to the addition of a direct flow link between the matrix and wellbore. Chen et al. (2016a, b) applied a numerical approach to study the flow behavior in a naturally fractured vuggy carbonate reservoir with large caves. The authors obtained pressure behavior using the Stehfest numerical inversion method. They observed that well radius affects the nature of the concavity and convexity of the curve.

Velazquez et al. (2005) proposed two models that describe the flow in naturally fractured vuggy carbonate reservoirs during transient and pseudo-steady-state interporosity flow. The first model considers flow from matrix and vugs to the fracture (triple-porosity/single-permeability), while the second model considers flow between interconnected vugs, in addition, to flow from matrix and vugs to fractures (triple-porosity/dual-permeability). The derivation of PDE for the triple-porosity/dual-permeability model presented by Velazquez et al. (2005) is shown hereunder.

The equation describing flow in the fracture system is given by

$\kappa \frac{1}{r_{\mathrm{D}}} \frac{\partial}{\partial r_{\mathrm{D}}}\left(r_{\mathrm{D}} \frac{\partial p_{\mathrm{Df}}}{\partial r_{\mathrm{D}}}\right)+\lambda_{\mathrm{mf}}\left(p_{\mathrm{Dm}}-p_{\mathrm{Df}}\right)+\lambda_{\mathrm{vf}}\left(p_{\mathrm{Dv}}-p_{\mathrm{Df}}\right)=\omega_{\mathrm{f}} \frac{\partial p_{\mathrm{Df}}}{\partial t_{\mathrm{D}}}$

while that describing flow in the matrix blocks is where

$\lambda_{\mathrm{mf}}=\sigma_{\mathrm{mf}} k_{\mathrm{m}} r_{\mathrm{w}}^{2} /\left(k_{\mathrm{f}}+k_{v}\right), \quad \lambda_{\mathrm{mv}}=\sigma_{\mathrm{mv}} k_{\mathrm{m}} r_{\mathrm{w}}^{2} /\left(k_{\mathrm{f}}+k_{\mathrm{v}}\right)$,

$\lambda_{\mathrm{vf}}=\sigma_{\mathrm{vf}} k_{\mathrm{vf}} r_{\mathrm{w}}^{2} /\left(k_{\mathrm{f}}+k_{\mathrm{v}}\right), \quad \kappa=k_{\mathrm{f}} /\left(k_{\mathrm{f}}+k_{\mathrm{v}}\right)$,

$t_{\mathrm{D}}=\left(k_{\mathrm{f}}+k_{\mathrm{v}}\right) t / \phi_{\mathrm{f}}\left(k_{\mathrm{f}}+k_{\mathrm{v}}\right), \quad \omega_{\mathrm{f}}=\phi_{\mathrm{f}} c_{\mathrm{f}} /\left(\phi_{\mathrm{f}} c_{\mathrm{f}}+\phi_{\mathrm{m}} c_{\mathrm{m}}+\phi_{\mathrm{v}} c_{\mathrm{v}}\right)$, and

$\omega_{\mathrm{v}}=\phi_{\mathrm{v}} c_{\mathrm{v}} /\left(\phi_{\mathrm{f}} c_{\mathrm{f}}+\phi_{\mathrm{m}} c_{\mathrm{m}}+\phi_{\mathrm{v}} c_{\mathrm{v}}\right)$.

In the case of the triple-porosity/single-permeability model, $k_{\mathrm{f}}$ and $k_{\mathrm{v}}$ are set to zero (i.e., $\kappa=1$ ) except in $\lambda_{\mathrm{vf}}$. The Laplace-space solutions to the triple-porosity/singlepermeability model are

$\overline{p_{\mathrm{Dv}}}=\overline{p_{\mathrm{Df}}}\left[\left(b_{1}+b_{2} u\right) /\left(b_{3}+b_{4} u+b_{4} u^{2}\right)\right]$

and

$\overline{p_{\mathrm{Dm}}}=\overline{p_{\mathrm{Df}}}\left(\frac{c_{1}+u c_{2}+u^{2} c_{3}}{d_{1}+u d_{2}+u^{2} d_{3}+u^{3} d_{4}}\right)$

Applying boundary conditions, the solution at the wellbore is given by

$\overline{p_{\mathrm{wD}}}=\frac{K_{0}[\sqrt{g(u)}]+s \sqrt{g(u)} K_{1}[\sqrt{g(u)}]}{u \sqrt{g(u)} K_{1}[\sqrt{g(u)}]+C_{\mathrm{D}} u\left\{K_{0}[\sqrt{g(u)}]+s \sqrt{g(u)} K_{1}[\sqrt{g(u)}]\right\}}$

For the no-flow boundary condition,

$\bar{p}_{\mathrm{Df}}=\frac{K_{1}\left[\sqrt{g(u)} r_{\mathrm{eD}}\right] I_{0}\left[\sqrt{g(u)} r_{\mathrm{D}}\right]+I_{1}\left[\sqrt{g(u)} r_{\mathrm{eD}}\right] K_{0}\left[\sqrt{g(u)} r_{\mathrm{D}}\right]}{u \sqrt{g(u)}\left\{K_{1}[\sqrt{g(u)}] I_{1}\left[\sqrt{g(u)} r_{\mathrm{eD}}\right]-I_{1}[\sqrt{g(u)}] K_{1}\left[\sqrt{g(u)} r_{\mathrm{eD}}\right]\right\}}$

and

$\bar{p}_{\mathrm{wD}}=\frac{1}{u^{2} \bar{q}_{\mathrm{wD}}}$

The Laplace-space solutions to the triple-porosity/dualpermeability model are

$\begin{aligned} \bar{p}_{\mathrm{Df}} & =\lambda_{1} A_{1} K_{0}\left(\alpha_{1} r_{\mathrm{D}}\right)+\lambda_{2} A_{2} K_{0}\left(\alpha_{2} r_{\mathrm{D}}\right) \\ & +\lambda_{3} B_{1} K_{0}\left(\alpha_{1} r_{\mathrm{D}}\right)+\lambda_{4} B_{2} K_{0}\left(\alpha_{2} r_{\mathrm{D}}\right)\end{aligned}$

and 


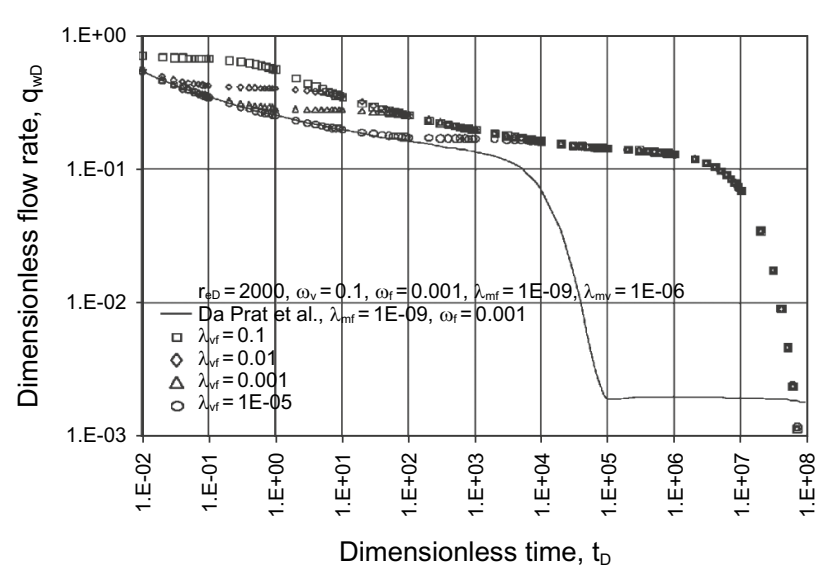

Fig. 11 Vuggy reservoir plot for dual-porosity (Velazquez et al. 2005)

$$
\bar{p}_{\mathrm{Dv}}=\lambda_{1} K_{0}\left(\alpha_{1} r_{\mathrm{D}}\right)+\lambda_{2} K_{0}\left(\alpha_{2} r_{\mathrm{D}}\right)+\lambda_{3} I_{0}\left(\alpha_{1} r_{\mathrm{D}}\right)+\lambda_{4} I_{0}\left(\alpha_{2} r_{\mathrm{D}}\right)
$$

The pressure response for the triple-porosity/single-permeability (unconnected vugs) model is shown in Fig. 10 for different values of $\lambda_{\mathrm{mf}}, \lambda_{\mathrm{vf}}, \lambda_{\mathrm{mv}}, \omega_{\mathrm{f}}$ and $\omega_{\mathrm{v}}$. A semi-log straight line appears at the early-time period which points to a fracture-controlled flow. Also, a similar semi-log straight line appears at the late time indicating a homogenous flow in the system components (fractures, vugs and matrix) where pressure is the same. The figure also shows anomalous slopes in the transient period with a slope ratio of around 2:1 of the early and late time. These slopes appear in this segment due to the presence of vugs. Also, these slopes are functions of $\lambda_{\mathrm{vf}} / \lambda_{\mathrm{mf}}$ and $\lambda_{\mathrm{mv}} / \lambda_{\mathrm{mf}}$.

Velazquez et al. (2005) also examined the analytical decline curves of unconnected vugs at different values of $\lambda_{\mathrm{mf}}, \lambda_{\mathrm{vf}}, \lambda_{\mathrm{mv}}, \omega_{\mathrm{f}}$ and $\omega_{\mathrm{v}}$ in comparison with the doubleporosity model proposed by Da Prat et al. (1981). The authors found significant differences between unconnected vugs and double-porosity behavior especially in the transient period as shown in Fig. 11. In some cases, the differences can also be observed in the boundary dominated periods.

Unlike the case of unconnected vugs, the authors did not observe a straight line in the semi-log plot during the early transient period. The difference in pressure responses during the transient period becomes larger at bigger $\omega_{\mathrm{f}}$ values. However, the straight line is observed in the late time once the homogenous flow is attained. The authors also compared the rate responses of triple-porosity/single-permeability, triple-porosity/dual-permeability and double-porosity/doublepermeability model of Da Prat et al. (1981). It was observed that differences in the rate occurred between the triple and double-porosity models during the boundary-dominated period, while differences in rate between the single and

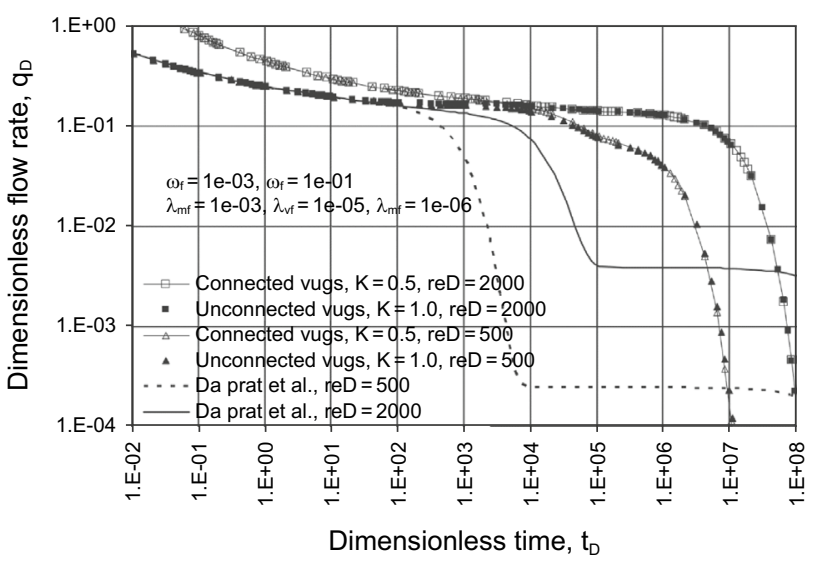

Fig. 12 Single and dual permeability is observed in the transient segment (Velazquez et al. 2005)

dual-permeability models were observed in the transient period (Fig. 12).

Although Velazquez et al. (2005) included the skin factor of a system in the transient flow solution of connected vugs, they did not discuss it in detail. The analysis of skin effect is important to realistically model production decline.

Nie et al. (2012) studied the pressure transient analysis of a naturally fractured vuggy reservoir with triple-porosity using a quadratic pressure gradient term. For an isotropic system, the diffusivity equation with the quadratic pressure gradient term included is

$\frac{1}{r} \frac{\partial}{\mathrm{d} r}\left(r \frac{\mathrm{d} p}{\mathrm{~d} r}\right)+C_{\mathrm{p}}\left(\frac{\mathrm{d} p}{\mathrm{~d} r}\right)^{2}=\mu \phi C_{\mathrm{t}} \frac{\mathrm{d} p}{\mathrm{~d} t}$

The authors utilized a model that idealizes the naturally fractured reservoir matrix block and vugs as spherical and unsteady interporosity flow from the matrix to fracture as well as from vugs to fracture, with no discharge from the matrix to vugs (Fig. 13). The assumptions made include a slightly compressible single-phase fluid, isotropic reservoir, no fluid storage in fractures.

The partial differential equation of the vug system was given by the authors as

$\left(\frac{\partial^{2} p_{\mathrm{vD}}}{\partial r_{\mathrm{vD}}^{2}}+\frac{2}{r_{\mathrm{rD}}} \frac{\partial p_{\mathrm{vD}}}{\partial r_{\mathrm{vD}}}\right)+\beta\left(\frac{\partial p_{\mathrm{vD}}}{\partial r_{\mathrm{vD}}}\right)^{2}=\frac{15 \omega_{\mathrm{v}}}{\lambda_{\mathrm{v}} C_{\mathrm{D}}} \frac{\partial p_{\mathrm{vD}}}{\partial t_{\mathrm{D}}}$

Their findings showed that the shapes of the pressure derivative curves are affected by the interporosity flow factor from vugs to fracture $\left(\lambda_{\mathrm{v}}\right)$ and interporosity flow factor from the matrix to fracture $\left(\lambda_{\mathrm{m}}\right)$. Similarly, the value of $\lambda$ controls the concave-shape observed in the later period of interporosity flow.

Jia et al. (2013) studied a vuggy-matrix double-porosity carbonate reservoir system in the Tahe oilfield of China. 


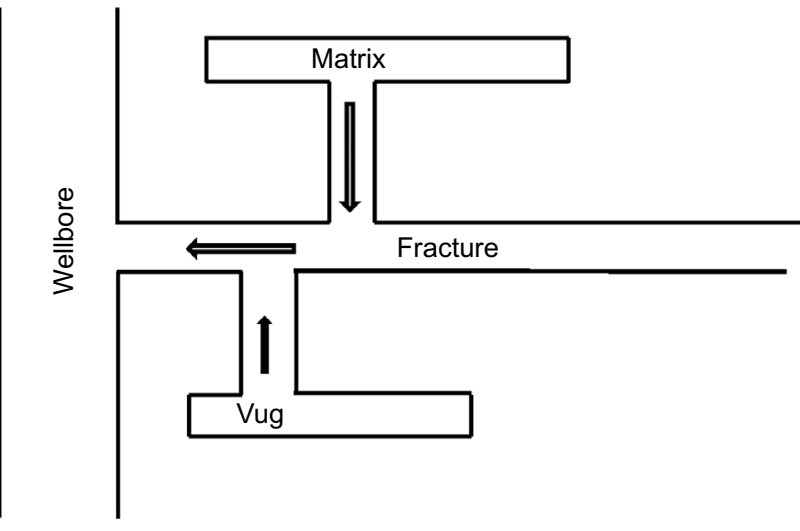

Fig. 13 Flow schematic of a porous vuggy reservoir (Jia et al. 2013)

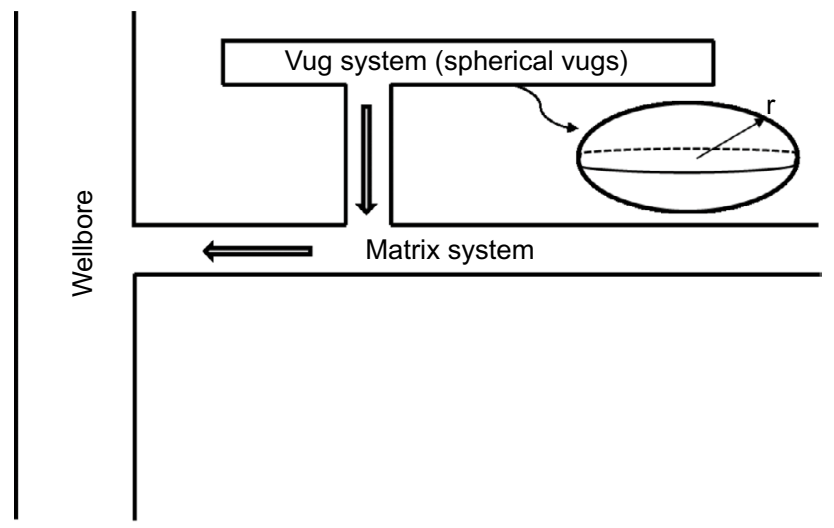

Fig. 14 Flow schematic of the porous vuggy reservoir (Jia et al. 2013)

Unlike other double-porosity models where a matrix-fracture continuum is considered, the model proposed by Jia et al. (2013) was developed with the assumption that the vugs are uniformly dispersed (not connect) and that vugsmatrix interporosity flow occurs under an unsteady state in non-fractured carbonate reservoirs. Thus, in such a model, fluid flow occurs from the vugs to the matrix and from the matrix to the wellbore as depicted in Fig. 14.

Several assumptions were made to formulate the model describing the flow such as single-phase flow behavior, homogenous and isotropic system, slightly compressible system. The equation governing flow in the matrix system (in a cylindrical coordinate system) is $\frac{\partial^{2} \bar{p}_{\mathrm{vD}}}{\partial r_{\mathrm{vD}}^{2}}+\frac{2}{r_{\mathrm{vD}}} \frac{\partial \bar{p}_{\mathrm{vD}}}{\partial r_{\mathrm{vD}}}=\frac{15 z \omega_{\mathrm{v}}}{\lambda_{\mathrm{v}}} \bar{p}_{\mathrm{vD}} \quad\left(0<r_{\mathrm{vD}}<1\right)$

The authors solved the dimensionless differential equations using Laplace transforms and inverted to real-space via Stephfest numerical inversion. This model was tested by matching real data to the log-log type-curves from the model.

\subsection{Pressure transient analysis for caves}

Although many well testing models have been developed for triple-porosity systems, such models lack a reasonable approximation of volumes and connectivity of large caves with the radius as big as tens of meters (Pang et al. 2019). Thus, Pang et al. (2019) developed a pressure transient model for a well that penetrates through a cave. The model introduces fluid flow and pressure wave propagation concept and diagnoses the cave's volume. Moreover, the model defines three parameters that affect the pressure response in the log-log plot. These parameters are wave coefficient, damping coefficient and cave shape factor. The model is given as follows.

Darcy flow in matrix outside wellbore:

$\frac{1}{r_{\mathrm{D}}} \frac{\partial}{\partial r_{\mathrm{D}}}\left(r_{\mathrm{D}} \frac{\partial p_{1 \mathrm{D}}}{\partial r_{\mathrm{D}}}\right)=\frac{\partial p_{1 \mathrm{D}}}{\partial t_{\mathrm{D}}}$

Darcy flow in the matrix outside the cave:

$\frac{1}{r_{\mathrm{D}}} \frac{\partial}{\partial r_{\mathrm{D}}}\left(r_{\mathrm{D}} \frac{\partial p_{2 \mathrm{D}}}{\partial r_{\mathrm{D}}}\right)=\frac{\partial p_{2 \mathrm{D}}}{\partial t_{\mathrm{D}}}$

The wellbore flow is modeled by

$p_{\mathrm{wfD}}=\left.\left(p_{1 \mathrm{D}}-s_{\mathrm{w}} r_{\mathrm{D}} \frac{\partial p_{1 \mathrm{D}}}{\partial r_{\mathrm{D}}}\right)\right|_{r_{\mathrm{D}}=1}$,

while the flow in the cave is modeled by

$p_{\mathrm{vD}}=\left.\left(p_{2 \mathrm{D}}-s_{\mathrm{v}} r_{\mathrm{D}} \frac{\partial p_{2 \mathrm{D}}}{\partial r_{\mathrm{D}}}\right)\right|_{r_{\mathrm{vD}}}$

The inner boundary condition is given by

$\frac{\partial^{2} \bar{p}_{\mathrm{mD}}}{\partial r_{\mathrm{mD}}^{2}}+\frac{1}{r_{\mathrm{mD}}} \frac{\partial \bar{p}_{\mathrm{mD}}}{\partial r_{\mathrm{mD}}}-\left.\frac{1}{5} \lambda_{\mathrm{vm}} \exp (-2 s)\left(\frac{\partial \bar{p}_{\mathrm{vD}}}{\partial r_{\mathrm{vD}}}\right)\right|_{r_{\mathrm{vD}}=1}=z \omega_{\mathrm{m}} \exp (-2 s) \bar{p}_{\mathrm{mD}}$

while that governing flow in the vug system (in a spherical coordinate system) is

$1=C_{\mathrm{wD}} \frac{\partial p_{\mathrm{wfD}}}{\partial t_{\mathrm{D}}}+C_{\mathrm{vD}} \frac{\partial p_{\mathrm{vD}}}{\partial t_{\mathrm{D}}}-\left[\left.\lambda \frac{\partial p_{1 \mathrm{D}}}{\partial r_{\mathrm{D}}}\right|_{r_{\mathrm{D}}=1}+\left.(1-\lambda) r_{\mathrm{vD}} \frac{\partial p_{2 \mathrm{D}}}{\partial r_{\mathrm{D}}}\right|_{r_{\mathrm{vD}}}\right]$

Outer boundary: 


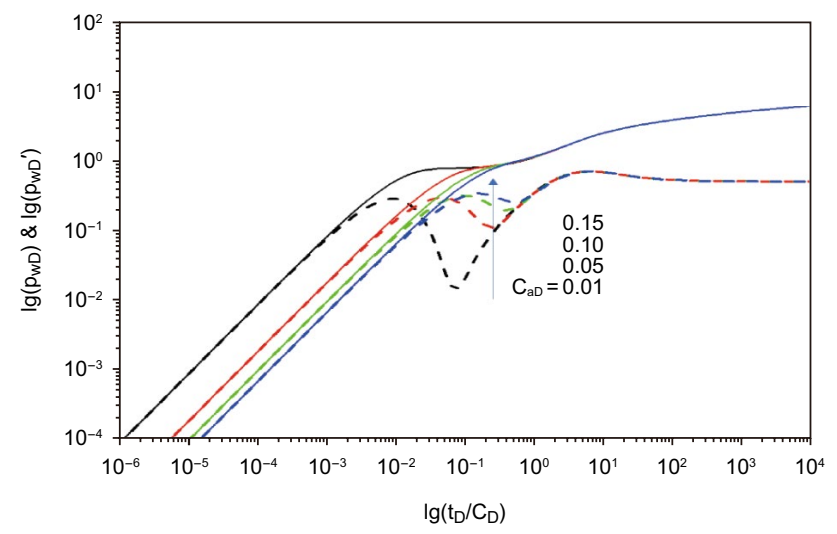

Fig. 15 Impact of wave coefficient on the pressure response (Ye et al. 2016)

$\left.\frac{\partial \overline{p_{1 \mathrm{D}}}}{\partial r_{\mathrm{D}}}\right|_{r_{\mathrm{eD} 1}}=\left.\frac{\partial \overline{p_{2 \mathrm{D}}}}{\partial r_{\mathrm{D}}}\right|_{r_{\mathrm{eD} 2}}=0$

Also, the relation between dimensionless bottomhole pressure and dimensionless cave pressure is

$\overline{p_{\mathrm{wfD}}}=\overline{p_{\mathrm{vD}}}-C_{\mathrm{pD}} t_{\mathrm{D}}^{\alpha-1} \mathrm{e}^{-C_{\mathrm{aD}} t_{\mathrm{D}}}$

where $C_{\mathrm{aD}}$ is the dimensionless wave coefficient that describes the wave flow in caves while $C_{\mathrm{pD}}$ is the dimensionless damping coefficient which describes pressure drop in caves. Also, $\alpha$ is the cave shape factor that accounts for the difference in shape between the actual cave and an ideal cylindrical cave. The authors obtained the solution to the set of equations in (56)-(61) in the Laplace domain. Using this solution, the authors studied the effect of key parameters on the $\log -\log$ pressure and pressure derivative-type curves. The plot was divided into four regions starting with wellbore storage followed by a transition period from wellbore storage to cave storage, full cave storage stage and finally, the formation-dominated transient flow. Figure 15 shows that the

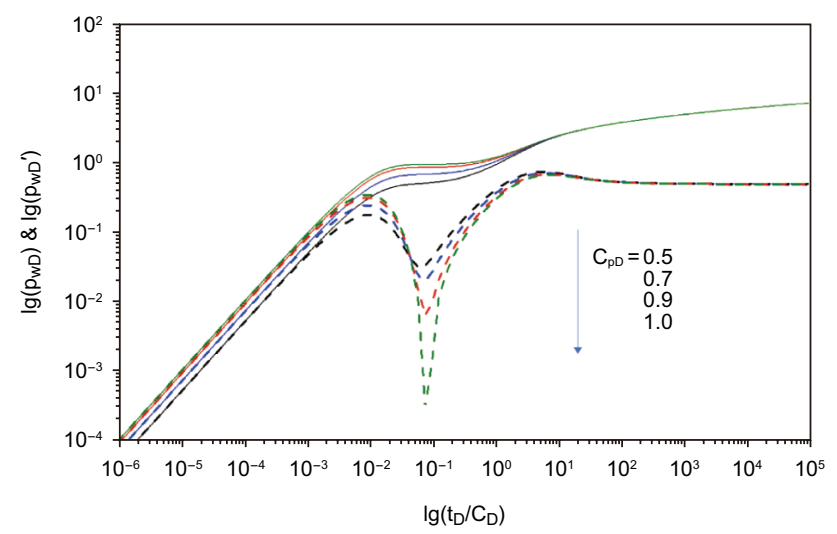

Fig. 16 Influence of wave coefficient on wellbore storage (Ye et al. 2016)

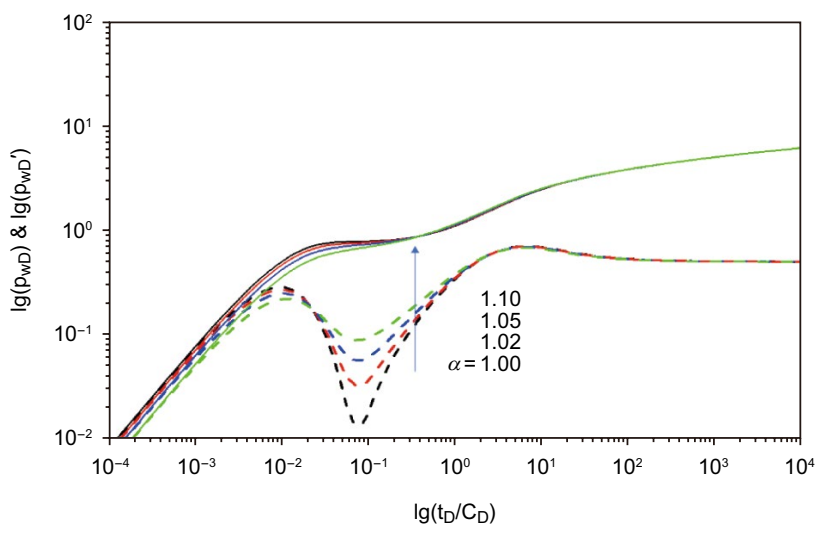

Fig. 17 Dimensionless plot with the effect of the shape factor (Ye et al. 2016)

wave coefficient affects the pressure response. The higher the $C_{\mathrm{aD}}$ the longer wellbore storage effect and subsequently the later wellbore/cave storage transition. Also, the bigger the $C_{\mathrm{aD}}$ the less pressure drop is observed in the full cave storage period. The matrix-dominated transient flow is the only segment that is not affected.

The effect of $C_{\mathrm{pD}}$ on the wellbore storage period is lesser than that of $C_{\mathrm{aD}}$ (Fig. 16). $C_{\mathrm{pD}}$ also affects the sharpness of the trough seen on the pressure derivative curve beyond the wellbore storage period. It is observed that the time at which the trough appears is the same for all values of $C_{\mathrm{pD}}$ as shown in Fig. 16. Finally, the effect of the shape factor $\alpha$ on the pressure and pressure derivative curves is shown in Fig. 17. It is observed that the shape factor affects the cave storage period.

\section{Field applications}

Olarewaju (1997) applied pressure transient analysis to the Hanifa reservoir to provide insight into the abnormally high flow rates recorded by the flow meters. Also, the permeability derived from well test was observed to be 40 times greater than the permeability determined from core plugs. To resolve this, the author applied analytical pressure transient analysis to confirm the existence of naturally fractures. The findings confirmed the existence of a dual-permeability system in place. The author further asserted that the use of a dual-porosity model to analyze this reservoir will lead to error in interpretation, as significant portions of the reservoir have little or no fracture and the matrix block contributes to the production. 


\section{Comparison of different pressure transient analysis models}

\subsection{Naturally fractured reservoirs}

In this section, analysis is made of the differences and similarities between the models developed for naturally fractured reservoirs, with emphasis laid on the applications of the models. It appears that in the early days of well test analysis, the geological model of a reservoir received much attention. For instance, Baker (1955) presented a method based on a geological model in which natural fissures of great extent characterized the formation. Pollard (1959) method was as a result of the fact that, from a geological point of view, the reservoir void space consisted of two types of voids. In a way, it was a pragmatic approach to solving the problem. However, the drawback of this approach was that it seems to apply only to that geological model. On the other hand, from a production viewpoint, Barenblatt et al. (1960) are the first to present a mathematical fluid flow model to describe flow dynamics in fissured formations based on fundamental laws of fluid mechanics. In his model, a homogeneous parallel system was set up to mimic primary porosity and fissures. This captured adequately the concept of property averaging as Darcy law is said to be valid for both the matrix and fissures in this setup. The Barenblatt et al. (1960) theory served as the fundamental basis upon which the Warren and Root (1963) model were developed, and their model accounting for different fractured reservoir types, with fluids either in the matrix or fissures.

The nature of the interaction between the matrix and the fissures is reflected by $\omega$ and $A$. However, the introduction of $w$ and $X$ to describe the fracture types has been a subject open to so many criticisms for years. Also, the presence of two parallel lines on the buildup test has received criticism as well for the wellbore storage that could have obscured the semi-log straight line. However, with the development of type curves, this concern was overcome.

Bourdet and Gringarten (1980) presented a method in which $w$ and $X$ can be determined by type-curve matching techniques, showing a practical and applicable method that can be used to analyze fractured reservoir's pressure buildup test. Warren and Root's model has been studied by several authors (Mavor and Ley 1979; Kazemi 1969; Bourdet and Gringarten 1980). The model has been extended to include transient interporosity flow and wellbore storage effects. According to the literature, it can be said that Warren and Root's model laid the foundation for many practical applications. The development of models by Barenblatt et al. (1960) and Warren and Root (1963) was based on the internal source concept. Kazemi (1969) and Streltsova (1984) models are conceptually different models. These authors utilized a multilayered system for fracture modeling and solved the system of equations, with the source as a boundary condition which shows that there is no basis for comparison between Kazemi (1969), Streltsova's model and Warren and Root (1963). Speaking from a geological viewpoint, a multilayer system is quite different from a vuggy, fissured/fractured one-layer reservoir, due to high permeability contrast.

From the practical viewpoint, the answer to the question of which model to use lies in the integration of pressure with geological models. An approach suggested by Ramey and Agarwal (1972) was to start with a simple homogenous system with conventional analysis and then build up from there as a basis to enable the achievement of pragmatic analysis.

\subsection{Vuggy reservoirs}

\subsubsection{Triple-porosity model}

To model fluid dynamics in vuggy reservoirs, reference is often made to models for naturally fractured reservoirs, such as dual-porosity and triple-porosity models and their extensions. The dual-porosity model was first developed by Barenblatt et al. (1960). Subsequently, Warren and Root proposed a complete dual-porosity model that has gained acceptance in naturally fractured reservoir modeling (Warren and Root 1963). Additionally, these models focused on the fluid flow between formation matrix and fractures (Kazemi et al. 1976; Coats 1989; Ueda et al. 1989; Saidi 1987; Thomas et al. 1983). Based on the classical dualporosity model, a model which subdivided the fracture grids into sections and accounting for the multiple interactions was proposed (Wu and Pruess 1988; Wu et al. 2011).

In the development of models for vuggy reservoirs, flow behavior was encountered which could not be explained by the dual-porosity model. Thus, the triple-porosity model was proposed, and this has found application in several fields. The triple-porosity effect has been shown by several authors (Yao et al. 2010) to be distinguishable in well test and has recently been extended by Kang et al. (2006) and Wu et al. (2011). The triple-porosity model can account for mass exchange between matrix, fracture and vug systems as well as the preferential flow phenomena. However, the triple-porosity model does not account for full-physics and thus is still an approximation to the actual flow behavior of naturally fractured vuggy systems.

\subsubsection{Equivalent-medium model}

This concept is different from the dual and triple-porosity models in that it assumes a fracture-vuggy reservoir as a continuous porous media with its heterogeneity represented by equivalent parameters. This model is simple and has a far-reaching application in rock hydraulics (Yao et al. 2010), 
with high calculation efficiency. Single-phase flow has its theoretical basis established over the decades with limited theories that account for multiphase flow and calculated the equivalent properties such as permeability and capillary curves (Yao et al. 2010; Kang et al. 2006). The theoretical basis for the equivalent flow model is aimed at reducing the order of the flow governing equation on a fine scale which is pivoted on scale theory. Thus, this is a macroscopic approach that reduces the heterogeneity effect and may lead to loss of signatures of vugs and fractures. Jia et al. (2013) presented an oversampling methodology to account for the macroscopic heterogeneity and connectivity in fracturevuggy mediums at a coarse grid scale. His model proved to be more accurate and efficient as compared to the equivalent-medium model. However, the model showed significant error in multiphase flow prediction due to its failure to capture the physics of flow at a fine scale in vugs and fractures (Yao et al. 2010).

\subsubsection{Discrete medium model}

Discrete surfaces of varying properties may result from a long period of geologic processes on carbonate rocks. A discrete vuggy system is often a result of washouts at varying periods. Thus, it is presumed that all formations growing with vugs are discrete and are categorized under the discrete medium model. This concept was first proposed for rock hydraulics by Snow (1969). However, what is currently used is a model proposed by Noorishad and Mehran (1982). The authors utilized the upstream technique to solve a 2D-solute diffusivity problem using finite-element analysis. In their approach, 2D and 1D surfaces were used for rock and fracture, respectively, with both of these coupled using the principle of superposition. However, the model had limited application in the petroleum industry prior to 1999, when it was applied to simulate a twophase flow (Kim and Deo 1999). Since then, several methods (Galerkin finite-element method; finite-volume method; finite-difference method, etc.) have been proposed and have served as an extension of the discrete model technique (Yao et al. 2010). Explicit description of vugs has been achieved by the discrete model using flux equivalent principal considering flows in fractures as seepages. These models have a good representation of reality as well as high precision and can be used to solve dual and triple-porosity problems as well as the equivalent-medium problems. Pulido et al. (Pulido et al. 2011) developed a similar approach with the inclusion of skin factor impact analysis. Additionally, Velazquez et al. (2005) made no distinction between channel, vugs or caves. The authors considered all additional porosity generated by dissolution in carbonate as vuggy porosity. This assumption may not be valid for caves with a large radius.

\section{Conclusion and recommendations}

In this work, we considered various methods describing highly heterogeneous forms of reservoir formations, with classification into the naturally fractured reservoirs with and without vugs and caves serving as the basis for model analysis and comparison. In describing the pattern of fluid flow within these formations, modeling attempts have been made to use either the porosity or permeability types to correct the changes in the pressure diffusivity equations. Most of the models reviewed have pros and cons depending on the condition under which each model is applied. For example, to model flow in formations with natural fractures or vugs, it is important to characterize the different porosity or permeability systems due to the varying relationships between the properties of the multiple media that make up the reservoir. Furthermore, in modeling flow in naturally fractured mediums, only Warren and Roots model have found wide-spread applications in many formation types. Thus, most of the recent techniques of pressure transient analysis in naturally fractured and vuggy reservoirs are based on the Warren and Roots model.

Furthermore, models for vuggy reservoirs have been based on the models developed for naturally fractured reservoirs, with the resulting models classified into equivalent-medium models and discrete medium models. These models differ from the dual and triple-porosity models in their assumption of the vuggy system as a continuum. Explicitly, the choice of models highly depends on the uniqueness or peculiarity of the candidate reservoir in question, how much information is available and what inference can be made of its data. Research in this area is still very active as there are ongoing efforts to improve the interpretation of data from these formations.

Acknowledgements The authors would like to acknowledge the financial support received from the College of Petroleum Engineering and Geosciences at KFUPM through the project SF20006 toward the completion of this work.

Open Access This article is licensed under a Creative Commons Attribution 4.0 International License, which permits use, sharing, adaptation, distribution and reproduction in any medium or format, as long as you give appropriate credit to the original author(s) and the source, provide a link to the Creative Commons licence, and indicate if changes were made. The images or other third party material in this article are included in the article's Creative Commons licence, unless indicated otherwise in a credit line to the material. If material is not included in the article's Creative Commons licence and your intended use is not permitted by statutory regulation or exceeds the permitted use, you will need to obtain permission directly from the copyright holder. To view a copy of this licence, visit http://creativecommons.org/licenses/by/4.0/.

\section{References}

Allan J, Sun SQ. Controls on recovery factor in fractured reservoirs: lessons learned from 100 fractured fields. In: SPE Annual 
Technical Conference Exhibition Society of Petroleum Engineers; 2003. https://doi.org/10.2118/84590-MS.

Alsharhan AS, Nairn AEM. Sedimentary basins and petroleum geology of the Middle East. Amsterdam: Elsevier; 1997.

Areshev EG, Le Dong T, San NT, Shnip OA. Reservoirs in fractured basement on the continental shelf of southern Vietnam. J Pet Geol. 1992;15(4):451-64. https://doi.org/10.1111/j.1747-5457.1992. tb01045.x.

Bahrami H, Rezaee R, Hossain M. Characterizing natural fractures productivity in tight gas reservoirs. J Pet Explor Prod Technol. 2012;2(2):107-15. https://doi.org/10.1007/s13202-012-0026-X.

Baker WJ. Flow in fissured formations. World Petroleum Congress; 1955.

Barenblatt G, Zheltov I, Kochina I. Basic concepts in the theory of seepage of homogeneous liquids in fissured rocks [strata]. J Appl Math Mech. 1960;24(5):1286-303. https://doi.org/10.1016/00218928(60)90107-6.

Barros-Galvis N, Villaseñor P, Samaniego F. Analytical modeling and contradictions in limestone reservoirs: Breccias, vugs, and fractures. J Pet Eng. 2015;2015:1-28. https://doi. org/10.1155/2015/895786.

Berumen S, Tiab D. Interpretation of stress damage on fracture conductivity. J Pet Sci Eng. 1997;17(1-2):71-85. https://doi.org/10.1016/ S0920-4105(96)00057-5.

Bourbiaux B. Fractured reservoir simulation: a challenging and rewarding issue. Oil Gas Sci Technol IFP. 2010;65(2):227-38. https:// doi.org/10.2516/ogst/2009063.

Bourdet D, Gringarten AC. Determination of fissure volume and block size in fractured reservoirs by type-curve analysis. In: SPE Annual Technical Conference and Exhibition, 21-24 September, Dallas, Texas; 1980. https://doi.org/10.2118/9293-MS.

Bourdet D, Whittle TM, Douglas AA, Pirard VM. A new set of type curves simplifies well test analysis; 1983

Chen L, Liu Y, Zhu Z, Gao D, Cao W, Li Q. The discrete numerical models and transient pressure curves of fractured-vuggy units in carbonate reservoir. In: Proceedings of the Advances in Materials, Machinery, Electrical Engineering; 2017. https://doi.org/10.2991/ ammee-17.2017.100.

Chen P, Wang X, Liu H, Huang Y, Chen S. A pressure-transient model for a fractured-vuggy carbonate reservoir with large-scale cave. Geosyst Eng. 2016a;9328:1-8. https://doi.org/10.1080/12269 328.2015.1093965.

Chen P, Wang X, Liu H, Huang Y, Chen S, Zhang H. A pressuretransient model for a fractured-vuggy carbonate reservoir with large-scale cave. Geosyst Eng. 2016b;19(2):69-76. https://doi. org/10.1080/12269328.2015.1093965.

Chen Z, Liao X, Liu Q, Corporation CO, Wang L, Technology E, et al. Pressure transient analysis in fractured horizontal wells with fracture networks. In: In: SPE Western Regional Meeting, 23-26 April, San Jose, California, USA; 2019. https://doi. org/10.2118/195286-MS.

Choquette PW, Pray LC. Geologic nomenclature and classification of porosity in sedimentary carbonates. Am Assoc Pet Geol Bull. 1970;54(2):207-50. https://doi.org/10.1306/5D25C98B-16C111D7-8645000102C1865D.

Cinco-Ley H, Samaniego FV, Kucuk F. The pressure transient behavior for naturally fractured reservoirs with multiple block size. In: SPE Annual Technical Conference and Exhibition, 22-26 September, Las Vegas, Nevada; 1985. https://doi.org/10.2118/14168-MS.

Coats KH. Implicit compositional simulation of single-porosity and dual-porosity reservoir. In: SPE Symposium on Reservoir Simulation, 6-8 February, Houston, Texas; 1989. https://doi. org/10.2118/18427-MS

Da Prat G, Cinco-Ley H, Ramey HJ. Decline curve analysis using type curves for two-porosity systems. SPE J. 1981;21(3):354-62. https ://doi.org/10.2118/9292-PA. de Swaan OA. Analytic solutions for determining naturally fractured reservoir properties by well testing. Soc Pet Eng AIME J. 1976;16(3):117-22. https://doi.org/10.2118/5346-PA.

Dikkers SAJ. Development history of the La Paz field. Venez J Inst Pet; 1964.

Engler TW, Tiab D. Interpretation of pressure tests in naturally fractured reservoirs without type curve matching. In: Permian Basin Oil and Gas Recovery Conference, 27-29 March, Midland, Texas; 1996. https://doi.org/10.2118/35163-MS.

Escobar FH, Tiab D. PEBI grid selection for numerical simulation of transient tests. In: SPE Western Regional/AAPG Pacific Section Joint Meeting, 20-22 May, Anchorage, Alaska; 2002. https://doi. org/10.2118/76783-MS.

Firoozabadi A. Recovery mechanisms in fractured reservoirs and field performance. J Can Pet Technol 2000;39(11). https://doi. org/10.2118/00-11-DAS

Friedman M, Teufel LW, Morse JD. Strain and stress analyses from calcite twin lamellae in experimental buckles and faulted drape-folds. Philos Trans R Soc A Math Phys Eng Sci 1976;283(1312):87107. https://doi.org/10.1098/rsta.1976.0071.

Fritsen A, Corrigan T. Establishment of a geological fracture model for dual porosity simulations on the Ekofisk field. In: Buller AT, Berg E, Hjelmeland O, Kleppe J, Torsæter O, Aasen JO (eds) North sea oil and gas reservoirs-II. Springer, Dordrecht. Springer, Netherlands. 1990. p. 173-84 https://doi. org/10.1007/978-94-009-0791-1_14.

Fuentes-Cruz G, Camacho-Velazquez R, Vasquez-Cruz M. Pressure transient and decline curve behaviors for partially penetrating wells completed in naturally fractured-vuggy reservoirs. In: SPE International Petroleum Conference in Mexico, 7-9 November, Puebla Pue., Mexico; 2004. https://doi.org/10.2118/92116-MS.

Gong J, Rossen WR. Modeling flow in naturally fractured reservoirs: effect of fracture aperture distribution on dominant sub-network for flow. Pet Sci. 2017;14(1):138-54. https://doi.org/10.1007/ s12182-016-0132-3.

Guo J, Nie R, Jia Y. Dual permeability flow behavior for modeling horizontal well production in fractured-vuggy carbonate reservoirs. J Hydrol. 2012;464-465:281-93. https://doi.org/10.1016/j.jhydr ol.2012.07.021

Harris JF, Taylor JL, Walper GL. Relation of deformation fractures in sedimentary rock to regional and local structures. Am Assoc Pet Geol. 1960;44(12):1853-73. https://doi.org/10.1306/0BDA6 257-16BD-11D7-8645000102C1865D.

Hermansen H, Thomas LK, Sylte JE, Aasboe BT. Twenty five years of Ekofisk reservoir management. In: SPE Annual Technical Conference and Exhibition, 5-8 October, San Antonio, Texas; 1997. https://doi.org/10.2118/38927-MS.

Hodgson RA. Regional study of jointing in Comb Ridge-Navajo Mountain Area, Arizona and Utah. Am Assoc Pet Geol Bull. 1961;45(1):1-38. https://doi.org/10.1306/0BDA6278-16BD11D7-8645000102C1865D.

Horner DR. Pressure build-up in wells. In: World Petroleum Congress; 1951.

Huitt JL. Fluid flow in simulated fractures. AIChE J. 1956;2(2):259-64.

Jia YL, Fan XY, Nie RS, Huang QH, Jia YL. Flow modeling of well test analysis for porous-vuggy carbonate reservoirs. Transp Porous Media. 2013;97(2):253-79. https://doi.org/10.1007/s1124 2-012-0121-y.

Kang Z, Wu YS, Li J, Wu Y, Zhang J, Wang G. Modeling multiphase flow in naturally fractured vuggy petroleum reservoirs. IN: SPE Annual Technical Conference and Exhibition, 24-27 September, San Antonio, Texas, USA; 2006. https://doi.org/10.2118/10235 6-MS.

Kazemi H. Pressure transient analysis of naturally fractured reservoirs with uniform fracture distribution. SPE J. 1969;9(4):451-62. https ://doi.org/10.2118/2156-A. 
Kazemi H, Merrill LS, Porterfield KL, Zeman PR. Numerical simulation of water-oil flow in naturally fractured reservoirs. Soc Pet Eng AIME J. 1976;16(6):317-26. https://doi.org/10.2118/5719-PA.

Kim JG, Deo MD. Comparison of the performance of a discrete fracture multiphase model with those using conventional methods. In: SPE Reservoir Simulation Symposium, 14-17 February, Houston, Texas; 1999. https://doi.org/10.2118/51928-MS.

Lamb H. Hydrodynamics. Dover Publications; 1945.

Levorsen A, Frederick B. Geology of petroleum. 2nd ed. San Francisco: W.H. Freeman and Co.; 1967.

Li XN, Shen JS, Yang WY, Li ZL. Automatic fracture-vug identification and extraction from electric imaging logging data based on path morphology. Pet Sci. 2019;16(1):58-76. https://doi. org/10.1007/s12182-018-0282-6.

Luthi SM. Fractured reservoir analysis BT — geological well logs: their use in reservoir modeling. In: Luthi SM, ed. Berlin, Heidelberg: Springer; 2001. p. 297-316.

Mavor MJ, Ley HL. Transient pressure behavior of naturally fractured reservoirs. In: SPE California Regional Meeting, 18-20 April, Ventura, California; 1979. https://doi.org/10.2118/7977-MS.

Najurieta HL. A theory for pressure transient analysis in naturally fractured reservoirs. J. Pet. Technol. 1980;32(07):1241-50. https://doi. org/10.2118/6017-PA.

Nelson RA. Geologic analysis of naturally fractured reservoirs. Gulf Professional Pub; 2001.

$\mathrm{Ng}$ MC, Aguilera R. Testing of horizontal gas wells in anisotropic naturally fractured reservoirs. In: SPE Annual Technical Conference and Exhibition, 6-9 October, Dallas, Texas; 1991. https:// doi.org/10.2118/22674-MS.

Nie R, Jia Y, Yu J, Liu B, Liu Y. The transient well test analysis of fractured-vuggy triple-porosity reservoir with the quadratic pressure gradient term. In: Latin American and Caribbean Petroleum Engineering Conference, 31 May-3 June, Cartagena de Indias, Colombia; 2009. https://doi.org/10.2118/120927-MS.

Nie R, Meng Y, Jia Y, et al. Dual porosity and dual permeability modeling of horizontal well in naturally fractured reservoir. Transp Porous Med. 2012;92:213-35. https://doi.org/10.1007/s1124 2-011-9898-3.

Noorishad J, Mehran M. An upstream finite element method for solution of transient transport equation in fractured porous media. Water Resour Res. 1982;18(3):588-96. https://doi.org/10.1029/ WR018i003p00588.

Odeh AS. Unsteady-state behavior of naturally fractured reservoirs. SPE J. 1965;5(1):60-6. https://doi.org/10.2118/966-PA.

Olarewaju JS. Pressure transient analysis of naturally fractured reservoirs: a case study. Middle East Oil Show and Conference, 15-18 March, Bahrain; 1997. https://doi.org/10.2118/37801-MS.

Ostensen RW. The effect of stress-dependent permeability on gas production and well testing. SPE Form Eval. 1986;1(03):227-35. https://doi.org/10.2118/11220-PA.

Owen D. Thomas LKSP. Ekofisk: first of the giant oil fields of western Europe: REPLY. AAPG Bull. 2002;68(65):195-224. https://doi. org/10.1306/03B59995-16D1-11D7-8645000102C1865D.

Pedrosa OA. Pressure transient response in stress-sensitive formations. In: SPE California Regional Meeting, 2-4 April, Oakland, CA; 1986. https://doi.org/10.2118/15115-MS.

Pirson R, Pirson S. Extension of the pollard analysis method of well pressure build-up and drawdown tests. In: 36th Annu. Fall Meet. Soc. Pet. Eng. AIME. Dallas, TX, USA; 1961. https://doi. org/10.2118/101-MS.

Pollard P. Evaluation of acid treatments from pressure build up analysis. Trans Am Inst Min Metall Pet Eng 1959;216:38-43. https:// doi.org/10.2118/981-G.

Price NJ. Fault and joint development in brittle and semi-brittle rock. Fault Jt. Dev. Brittle Semi-Brittle Rock. 1966.
Pulido H, Galicia-munoz G, Valdés-pérez AR, Díaz-garcia F, Nacional $\mathrm{U}$, México A De, et al. Improve reserves estimation using interporosity skin in naturally fractured reservoirs. In: Proceedings, Thirty-Sixth Workshop on Geothermal Reservoir Engineering Stanford University, Stanford, California, January 31-February 2, 2011 SGP-TR-191.

Raghavan R, Scorer JDT, Miller FG. An investigation by numerical methods of the effect of pressure-dependent rock and fluid properties on well flow tests. SPEJ. 1972;12(3):267-75. https://doi. org/10.2118/2617-PA.

Ramey HJ, Agarwal RG. Annulus unloading rates as influenced by wellbore storage and skin effect. SPE J. 1972;12(5):453-62. https ://doi.org/10.2118/3538-PA.

Rezk MG. Analysis of pressure transient tests in naturally fractured reservoirs. Oil Gas Res OMICS Int. 2016;02(03):1-10. https:// doi.org/10.4172/2472-0518.1000121.

Saidi AM. Reservoir engineering of fractured reservoirs: fundamental and practical aspects. Paris: Total; 1987.

Samaniego VF, Cinco-Ley H, Montiel HD. Evaluation of naturally fractured gas reservoirs through pressure transient testing. In: SPE Annual Technical Conference and Exhibition, 22-26 September, Las Vegas, Nevada; 1985. https://doi.org/10.2118/14359-MS.

Snow D. Anisotropic permeability of fractured media. Water Resour Res. 1969;5(6):1273-89. https://doi.org/10.1029/WR005i006p 01273.

Streltsova TD. Buildup analysis for interference tests in stratified formations. J Pet Technol. 1984;36(2):301-10. https://doi. org/10.2118/10265-PA.

Tandom P, Ngoc N, Tjia H, Lloyd P. Identifying and evaluating producing horizons in fractured basement. In: SPE Asia Pacific Improv. Oil Recover. Conf. Society of Petroleum Engineers; 1999. https ://doi.org/10.2118/57324-MS.

Thomas LK, Dixon TN, Pierson RG. Fractured reservoir simulation. Soc Pet Eng J. 1983;23(1):42-54. https://doi. org/10.2118/9305-PA.

Tiab D. Analysis of pressure and pressure derivative without typecurve matching: vertically fractured wells in closed systems. J Pet Sci Eng. 1994;11(4):323-33. https://doi.org/10.1016/09204105(94)90050-7.

Ueda Y, Murata S, Watanabe Y, Funatsu K. Investigation of the shape factor used in the dual-porosity reservoir simulator. In: SPE AsiaPacific Conference, 13-15 September, Sydney, Australia; 1989. https://doi.org/10.2118/SPE-19469-MS.

Unam P, Pemex E. Behavior in naturally fractured vuggy carbonate reservoirs. Engineering; 2005.

van Golf-Racht TD. Fundamentals of fractured reservoir engineering. Amsterdam: Elsevier; 1982.

Velazquez RC, Vasquez-Cruz MA, Castrejon-Aivar R, Arana-Ortiz V. Pressure transient and decline curve behaviors in naturally fractured vuggy carbonate reservoirs. SPE Reserv. Eval. Eng. 2005;8:95-112. https://doi.org/10.2118/77689-PA.

Warren JE, Root PJ. The behavior of naturally fractured reservoirs. SPE J. 1963;3(03):245-55. https://doi.org/10.2118/426-PA.

Wilkinson D, Hammond PS. A perturbation method for mixed boundary-value problems in pressure transient testing. Transp Porous Media. 1990;5(6):609-36.

Willis DG, Hubbert MK. Important fractured reservoirs in the United States (U.S.A.). In: 4th World Petroleum Congress, 6-15 June, Rome, Italy; 1955. World Petroleum Congress.

Wu YS, Pruess K. A multiple-porosity method for simulation of naturally fractured petroleum reservoirs. SPE Reserv Eng. 1988;3(1):327-36. https://doi.org/10.2118/15129-PA.

Wu YS, Di Y, Kang Z, Fakcharoenphol P. A multiple-continuum model for simulating single-phase and multiphase flow in naturally fractured vuggy reservoirs. J Pet Sci Eng. 2011;78(1):13-22. https:// doi.org/10.1016/j.petrol.2011.05.004. 
Xiong Y, Xiong W, Cai M, Hou C, Wang C. Petroleum. Amsterdam: Elsevier; 2016. http://dx.doi.org/10.1016/j.petlm.2016.09.004.

Yao J, Huang Z-Q. Fractured vuggy carbonate reservoir simulation. 2017. http://link.springer.com/10.1007/978-3-662-55032-8.

Yao J, Huang Z, Li Y, Wang C, Lv X. Discrete fracture-vug network model for modeling fluid flow in fractured vuggy porous media. In: International Oil and Gas Conference and Exhibition in China, 8-10 June, Beijing, China; 2010. https://doi.org/10.2118/13028 7-MS.
Ye Z, Chen D, Pan Z, Zhang G, Xia Y, Ding X. An improved Langmuir model for evaluating methane adsorption capacity in shale under various pressures and temperatures. J Nat Gas Sci Eng. 2016;31:658-80. https://doi.org/10.1016/j.jngse.2016.03.070.

Yilmaz O, Nolen-Hoeksema RC, Nur A. Pore pressure profiles in fractured and compliant rocks1. Geophys Prospect. 1994;42(6):693714. https://doi.org/10.1111/j.1365-2478.1994.tb00236.x. 